\title{
CALAMAGROSTIS (POACEAE, AGROSTIDINAE) IN VIETNAM
}

\author{
Beata Paszko ${ }^{1}$, Bing LiU \& Hai-Ying Ma
}

\begin{abstract}
New taxonomic and distributional data are provided for three eastern Asian species of Calamagrostis (Poaceae, Agrostidinae) in Vietnam: C. abnormis (Hook. f.) Shukla, C. extremiorientalis (Tzvel.) Prob. and C. tripilifera Hook. f. The new national record of $C$. extremiorientalis is reported here for Vietnam, where it was previously misidentified as $C$. elatior (Griseb.) A. Camus or Deyeuxia petelotii Hitchc. (= C. abnormis). The presence of C. tripilifera and C. abnormis in Vietnam is confirmed. Additional new provincial records of $C$. extremiorientalis from Japan's Bonin Islands (also known as the Ogasawara Islands) and C. tripilifera from Daba Mountains in the northern Chongqing Municipality (Central China) are reported here. A key for identification of Calamagrostis species in Vietnam, nomenclatural data and information about geographical, altitudinal and habitat distributions are provided. Maps showing their complete distribution in eastern Asia are presented.
\end{abstract}

Key words: Agrostis, Asia, Deyeuxia, distribution, Eastern Asian region, nomenclature, taxonomy

Beata Paszko, Department of Vascular Plants, W. Szafer Institute of Botany, Polish Academy of Sciences, Lubicz 46, 31-512 Kraków, Poland; e-mail: b.paszko@botany.pl

Bing Liu, State Key Laboratory of Systematic and Evolutionary Botany, Institute of Botany, Chinese Academy of Sciences, Beijing 100093, China

Hai-Ying Ma, School of Life Sciences, Yunnan University, Kunming 650091, China

\section{INTRODUCTION}

Recent molecular study of ribosomal and plastid DNA sequences indicates that Deyeuxia Clarion ex P. Beauv. is a synonym of Calamagrostis Adans. (Poaceae, Agrostidinae), and that many Eurasian species that have been variously treated in these genera are closely related (Saarela et al. 2010; J. M. Saarela, unpubl. data). In view of this, Paszko $(2015,2016)$ and Paszko et al. (2016a, b) have applied a more broadly defined concept of the genus Calamagrostis, including Asian species of Deyeuxia, in an ongoing project to compile a new treatment of Calamagrostis for Flora of Pan-Himalaya and Flora of Nepal in accordance with the molecular evidence.

Describing the geographical distribution of a species depends on correct identification of specimens. Unfortunately, misidentification of specimens at species level is frequent in herbarium materials and in the literature, particularly in difficult groups like grasses. The first author and her collaborators

\footnotetext{
1 Corresponding author
}

revealed several such cases in Calamagrostis and Deyeuxia (Paszko \& Ma 2011; Paszko 2014, 2015; Paszko \& Soreng 2013; Paszko et al. 2013, 2016a, b) as well as in Agrostis (Nobis et al. 2016; Paszko \& Liu 2016).

The aim of this study is to revise and update the nomenclature, taxonomy and geographical distribution of the Calamagrostis species occurring in Vietnam. Two species of the genus Calamagrostis, C. elatior (Griseb.) A. Camus and C. tripilifera Hook. f., were recorded from Indochina (comprising Vietnam, Laos and Cambodia) by Camus (1928) and later by Schmid (1958). Schmid (1958) added Aulacolepis petelotii Hitchc., nom. inval. [= C. abnormis (Hook. f. emend. Paszko) Shukla]. Vietnamese records of the Calamagrostis species are based on Petelot's gatherings, housed in herbarium $P$ and possibly elsewhere. All of them were collected by Petelot in northwestern Vietnam in the area of the Hoan Lien Mts, which extend south from the Ailao Shan Mts in China, the southeasternmost extension of the Himalayan chain. 
In Checklist of Plant Species in Vietnam, Khoi and Đo (2005) recorded three species of Agrostis (A. micrantha Steud., A. stolonifera L., A. triaristata Knapp), one species of Calamagrostis [C. elatior (Hook. f.) A. Camus; incorrect citation of basionym author] and one species of Deyeuxia [D. petelotii (Hitchc.) S. M. Phillips $\&$ W. L. Chen]. They placed Calamagrostis tripilifera Hook. f. in synonymy of Agrostis triaristata. Recently, Tran and Vu (2013) made a revision of species within the tribe Aveneae (Poaceae) for the flora of Vietnam. They followed Khoi and Đo's (2005) treatment, with one exception. Tran and $\mathrm{Vu}$ (2013) referred to D. petelotii specimens identified by Camus (1928) and Khoi and Đo (2005) as $C$. elatior.

In the course of revision of species of Calamagrostis and Agrostis in Vietnam, we found that Khoi and Đo (2005) and Tran and Vu (2013) invalidly applied Knapp's name $A$. triaristata, a species described from Europe, for specimens earlier identified by Camus (1928) and Schmid (1958) as Calamagrostis tripilifera. Khoi and Đo (2005) also incorrectly cited the basionym author for the name Calamagrostis elatior, as noted by Tran and $\mathrm{Vu}$ (2013); the correct authorship for this combination is C. elatior (Griseb.) A. Camus.

In the present paper, the misidentified species previously reported as C. elatior (Camus 1928; Schmid 1958; Khoi \& Đo 2005) is correctly named C. extremiorientalis (Tzvel.) Prob. We also confirmed the identification of Vietnamese $C$. tripilifera made by Camus (1928). The study of the overall distribution of these three Vietnamese species of Calamagrostis revealed the first record of $C$. tripilifera from the Daba Mts in northern Chongqing Municipality in southwest China, and the first record of C. extremiorientalis from Japan's Bonin Islands (also known as the Ogasawara Islands).

\section{MATERIAL AND METHODS}

We studied specimens of Calamagrostis abnormis, C. extremiorientalis and C. tripilifera from the following herbaria: BM, CAS, CDBI, E, GH, K, KRAM, KUN, L, LE, NEFI, P, PE, S, TI, US and W (Thiers 2016). All revised localities of Calamagrostis abnormis, C. extremiorientalis and $C$. tripilifera are cited. The overall geographical ranges of species are shown on dot maps (Figs 1-3) produced using SimpleMappr (http://www. simplemappr.net/). Geographic coordinates were acquired using Google Earth (http://www.google.com/ earth/download/ge/), Google Maps (www.google.com/ maps) and topographic maps (Vlasenko 2008). If this information was not part of the original collection data, estimated coordinates are given in square brackets. To compare some historical localities of $\mathrm{H}$. Handel-Mazzetti, J. D. Hooker and F. Kingdon-Ward with current geographic locations, we used their recollections (Hooker 1854; Handel-Mazzetti 1996; Kingdon-Ward 1936, 1940) and letters (Hooker 1847-1851). Other resources for place and geographical feature names were the maps compiled by Gören Herner of the routes of the Swedish botanist Karl August Harald (Harry) Smith (Herner 1988). Because all three species have complicated nomenclature, we provide complete synonymy. Diagnostic characteristics for species studied are given in Table 1.

\section{KeY TO THE SPECIES OF CALAMAGROSTIS IN VIETNAM}

1. Callus hairs longer than lemma, 1.26-2.25 $\times$ length of lemma; lemmas $0.39-0.61 \times$ length of lower glumes; rachilla prolongations frequently absent, sometimes present in the form of naked rachis ....

2. C. extremiorientalis

1. Callus hairs not more than $1 / 2$ as long as lemma, $0.2-0.5 \times$ length of lemma; lemmas $0.60-1.03 \times$ length of lower glumes; hairy rachilla prolongations always present $\ldots \ldots \ldots \ldots \ldots \ldots \ldots \ldots 2$

2. Lower glumes 1.9-2.6 mm long; lemmas 1.85$2.50 \mathrm{~mm}$ long; lemmas awnless or with short mucro, $0.15-0.33 \mathrm{~mm}$ long, inserted subapically; lack of lateral mucros on lemmas . . . . . . . . .

1. C. abnormis

2. Lower glumes 3.05-7.10 mm long; lemmas 2.65$5.50 \mathrm{~mm}$ long; lemmas always with well developed geniculate awn, 3.9-9.2 mm long, inserted at lower part of lemma; lateral mucros on lemmas almost always present, $0.20-0.85 \mathrm{~mm}$ long ......... 3. C. tripilifera

\section{TAXONOMIC TREATMENT}

\section{Calamagrostis abnormis (Hook. f.) Shukla}

Grasses of North-Eastern India: 45. 1996.

Basionym: Deyeuxia abnormis Hook. f., Fl. Brit. India 7(22): 268. 1896, s.str. emend. Paszko (in Paszko \& Soreng, Phytotaxa 111(1): 6. 2013). LeCtotyPe 
Table 1. Diagnostic characters for Calamagrostis abnormis (Hook. f.) Shukla, C. extremiorientalis (Tzvel.) Prob. and C. tripilifera Hook. f. The number of specimens examined is given in brackets below the species name.

\begin{tabular}{|c|c|c|c|}
\hline Species / Character & $\begin{array}{c}\text { C. abnormis } \\
\text { (33) }\end{array}$ & $\begin{array}{c}\text { C. extremiorientalis } \\
(120)\end{array}$ & $\begin{array}{c}\text { C. tripilifera } \\
(82)\end{array}$ \\
\hline Lower glume length (LGL) [mm] & $1.90-2.55$ & $4.25-7.65$ & $3.05-7.10$ \\
\hline Lower glume width (LGW) [mm] & $0.50-0.95$ & $0.65-1.10$ & $1.15-2.75$ \\
\hline Upper glume length [mm] & $1.90-2.63$ & $4.05-6.95$ & $2.75-6.40$ \\
\hline Upper glume width [mm] & $0.55-0.88$ & $0.6-1.1$ & $0.8-1.8$ \\
\hline Callus hair length (CHL) [mm] & $0.6-1.0$ & $3.75-5.85$ & $0.65-1.75$ \\
\hline Lemma length (LL) $[\mathrm{mm}]$ & $1.85-2.50$ & $2.3-3.5$ & $2.65-5.50$ \\
\hline Palea length [mm] & $1.40-1.85$ & $1.15-2.60$ & $1.85-3.80$ \\
\hline Rachilla length [mm] & $0.15-0.63$ & $0.05-5.00$ & $0.30-1.25$ \\
\hline Rachilla length with hairs [mm] & $0.6-1.5$ & $1.5-3.0$ & $1.5-3.0$ \\
\hline Anther length [mm] & $0.45-0.90$ & $0.9-1.9$ & $0.6-1.5$ \\
\hline Lemma awn length [mm] & $\begin{array}{l}\text { Lemmas awnless or with short } \\
\text { mucro } c a 0.15-0.33 \mathrm{~mm} \text { long, } \\
\text { inserted subapically }\end{array}$ & $0.75-3.20$ & $3.9-9.2$ \\
\hline $\begin{array}{l}\text { Awn insertion, measured as length from } \\
\text { base of lemma to point of awn insertion } \\
\text { (AI) }[\mathrm{mm}]\end{array}$ & - & $1.38-3.15$ & $0.35-1.50$ \\
\hline Length of lateral mucros at lemma tip [mm] & Lack of lateral mucros & Lack of lateral mucros & $0.20-0.85$ \\
\hline LL/LGL & $0.84-1.34$ & $0.39-0.61$ & $0.60-1.03$ \\
\hline $\mathrm{CHL} / \mathrm{LL}$ & $0.25-0.49$ & $1.26-2.25$ & $0.20-0.48$ \\
\hline LGW/LGL & $0.24-0.45$ & $0.11-0.20$ & $0.25-0.51$ \\
\hline $\mathrm{AI} / \mathrm{LL}$ & $0.86-0.98$ & $0.53-0.98$ & $0.10-0.41$ \\
\hline
\end{tabular}

(designated by Bor 1954a: 442): INDIA. MeghalaYA. [East Khasi Hills Distr.]: Khasia, Surureem [Serrarim, Sohra rin, Sohrarim] [25.349 $\left.{ }^{\circ} \mathrm{N}, 91.739^{\circ} \mathrm{E}\right]$, 26 June 1850, Hooker f. \& Thomson 1232 (Lестотуре K000032321!).

Aulacolepis petelotii Hitchc., J. Wash. Acad. Sci. 24(7): 291. 1934, nom. inval. Agrostis petelotii (Hitchc.) Noltie, Edinburgh J. Bot. 56(3): 386. 1999. Deyeuxia petelotii (Hitchc.) S. M. Phillips \& W. L. Chen, Novon 13(3): 319. 2003. Aniselytron petelotii (Hitchc.) Soják, Čas. Nár. Muz. Praze, Řada Prír. 148(3-4): 202. 1979 [1980]. Neoaulacolepis petelotii (Hitchc.) Rauschert, Taxon 31(3): 561. 1982. Calamagrostis petelotii (Hitchc.) Govaerts, World Checkl. Seed Pl. 3(1): 11. 1999. TYPE: VIETNAM. [LAo CAI]. [Sa Pa Distr.]: Tonkin, environs de Chapu [Chapa, Sa Pá, Sapa], chemin au dela du col de Lo Qui Ho, alt. ca $1900 \mathrm{~m}\left[22.303^{\circ} \mathrm{N}, 103.775^{\circ} \mathrm{E}\right]$, Aug. 1933, Pételot 4743 (HOLOTYPE US00130363!; ISOTYPES P00640971!, L0044027 (fragm. ex US). Synonymized by Korthof \& Veldkamp (1984).

Agrostis continentalis Hand.-Mazz., Symb. Sin. 7(5): 1297, pl. 40, f. 2. 1936. Deyeuxia continentalis (Hand.Mazz.) L. Liou, Vasc. Pl. Hengduan Mountain 2: 2240. 1994. LeCtotype (designated by Paszko \& Soreng
2013: 6): CHINA. YunNAN. [Yuanmou Co.]: in demissis subtropicis fluminis Djinscha-djiang ('Yangtse') ad boreo-occid. urbis Yünnanfu [Kunming], inter oppidum Yüenmou et vicum Hailo [between Yuanmou Town and Hailuocun Village], ad fossas [in ditch], alt. $1050-1350 \mathrm{~m}\left[25.855^{\circ} \mathrm{N}, 101.875^{\circ} \mathrm{E}\right], 10$ Oct. 1914 , Handel-Mazzetti 5018 (LECTOTYPE W1940-0014533!, ISOLECTOTYPES WU0061516!, WU0061517!).

Anisachne gracilis Keng, J. Wash. Acad. Sci. 48(4): 117-118, f. 2. 1958. Aniselytron gracilis (Keng) N. X. Zhao, J. Trop. \& Subtrop. Bot. 3(2): 50. 1995. LeCtoTYPE (designated by Paszko \& Soreng 2013: 6); the original blue-line illustration of Anisachne gracilis published in Keng (1958: 118, f. 2: 1-4) and in Lu et al. (2006: 475, f. 475) drawn based on type collection Hou 2143 (LeCtotype US!). Epitype (designated by Paszko \& Soreng 2013: 6): CHINA. YunNAN. Eastern slopes of Likiang Snow Range, Yangtze watershed $\left[27.103^{\circ} \mathrm{N}\right.$, 100.246 ${ }^{\circ} \mathrm{E}$ ], 1923-1924, Rock 10693 (EPITYPE US!). Synonymized by Korthof \& Veldkamp (1984).

Agrostis zenkeri auct. non Trin. (p.p.): Bor, Kew Bulletin 9: 441. 1954; Bor, Grasses Burma, Ceylon, India \& Pakistan: 392. 1960; Hara, Fl. Eastern Himalaya 1: 349. 1966; Rajbhandahari, J. Japan. Bot. 60: 77. 1985; 
Moulik, Grasses \& Bamboos India: 380. 1997; Press et al., Annot. Checkl. Fl. Pl. Nepal: 123. 2000.

Deyeuxia abnormis auct. non Hook.f. s.str. (p.p.): Phillips \& Chen, Novon 13: 319. 2003; Lu et al., Fl. China 22: 351. 2006.

A reliable description of Deyeuxia abnormis Hook. f. s.str. emend. Paszko was provided by Paszko and Soreng (2013). Schmid (1958) considered in the genus Aulacolepis Hack., nom. illeg. hom., a new species, Aulacolepis petelotii Hitchc., nom. inval., which should be treated within the genus Calamagrostis, as C. abnormis (Hook. f.) Shukla, based on Deyeuxia abnormis Hook.

f. emend. Paszko (in Paszko \& Soreng 2013). Aulacolepis petelotii was described by Hitchcock (1934: 291) based on Pételot's collection from Fan Si Pan (Fansipan) Mt., the highest point in Indochina (3,143 m). Mount Fan Si Pan is situated on the border between Lai Chau and Lao Cai Provinces, $9 \mathrm{~km}$ southwest of $\mathrm{Sa} \mathrm{Pa}$, a Vietnamese mountain health resort.

Paszko and Soreng (2013) amended the diagnosis and description of Hooker's Deyeuxia abnormis, rejected Noltie's (1999) relectotypification of D. abnormis, reverting to Bor's (1954: 442) original designation, and showed taxonomic relationships between $D$. abnormis and its allies. Currently, we treat it under the genus Calamagrostis in accordance with the molecular evidence (Saarela et al. 2010; J. M. Saarela, unpubl. data).

HABITAT AND PHENOLOGY. A perennial densely tufted grass that grows in open primary, humid, broadleaved and mixed evergreen forests (with Alnus nepalensis D. Don, Quercus semecarpifolia Sm., Pinus wallichiana A. B. Jacks., P. yunnanensis Franch.), grasslands, meadows, pastures and roadsides. 1200-3200 m. Flowers in JulySeptember.

General Distribution. Bhutan (Deothang, Thimphu, Punakha, Tongsa, Bumthang, Mongar and Tashigang Districts), China (W Guizhou and Yunnan Provinces), India [Arunachal Pradesh (currently under Indian administration, claimed by China as Zangnan, a region of Xizang Province), Manipur and Meghalaya Provinces], Nepal (Dolkha, Ramechhap, Sankhuwasabha and Solu
Khumbu Districts) and northwest Vietnam (Lao Kay Province) (Fig. 1).

Notes. The hitherto known geographical range of $C$. abnormis extends from central and eastern Nepal through most of the Eastern Himalayas in Bhutan and India (Arunachal Pradesh), through south central China (W Guizhou, Yunnan), extending into northwest Vietnam and into the northeastern Indian hill states in Manipur (Manipur Hills) and Meghalaya (Khasi Hills) (Noltie 1999, 2000; Paszko \& Soreng 2013). In China it is distributed mainly on the Yunnan-Guizhou Plateau, with some adjacent localities in the southeastern part of the Qinghai-Tibetan Plateau. In Vietnam it is recorded only from Mount Fan Si Pan (HandelMazzetti 1936, Schmid 1958). The gap between the localities at Manipur (NE India) and Yongde Co. (Yunnan, China) extends for $500 \mathrm{~km}$. The disjunct distribution records of this species may be due to lack of field work in areas on the southern slopes and the western part of the eastern slopes of the Himalayas, or the disjunct distribution pattern may be real.

Specimens examined: BHUTAN. Bumthang Distr.: Batpalathang, E side of river opposite Jakar, pasture, alt. $2650 \mathrm{~m}, 27.567^{\circ} \mathrm{N}, 90.75^{\circ} \mathrm{E}, 17 \mathrm{Sept} .1998$, Noltie et al. 232A (E); Kiki (Kyi Kyi) La, $9 \mathrm{~km} \mathrm{~S}$ of Jakar, among scrub in open Pinus wallichiana forest, alt. $2860 \mathrm{~m}, 27.533^{\circ} \mathrm{N}, 90.733^{\circ} \mathrm{E}, 5$ Sept. 1998 , Noltie et al. 86 (E); Beside Dhur Chu, Thangbi, edge of Pinus wallichiana forest, alt. $2700 \mathrm{~m}, 27.617^{\circ} \mathrm{N}, 90.717^{\circ} \mathrm{E}$, 17 Sept. 1998, Noltie et al. 237 (E). Mongar Distr.: Sengor, bare area at edge of car park in area of degraded pasture, alt. $3000 \mathrm{~m}, 27.367^{\circ} \mathrm{N}, 91.017^{\circ} \mathrm{E}, 16$ Sept. 1998 , Noltie et al. 221 (E). PunAKha Distr.: S side of pass between Nobding and Phubjikah, frequent in rough yak pasture with scattered dwarf bamboo, alt. $3100 \mathrm{~m}$, $27.504^{\circ} \mathrm{N}, 90.167^{\circ} \mathrm{E}, 24$ Sept. 1988 , J. R. I. Wood 6727 (E). Tashigang Distr.: Airstrip above Yonpu La, disturbed heathy grassland, with some young planted trees of Cryptomeria japonica, alt. $2500 \mathrm{~m}, 27.25^{\circ} \mathrm{N}$, $91.517^{\circ} \mathrm{E}, 9$ Sept. 1998 , Noltie et al. 159 (E). TнімPU DisTR.: between Motithang and Phajoding, bare earthen banks in cleared forest by path, alt. $2800 \mathrm{~m}\left[27.48^{\circ} \mathrm{N}\right.$, $89.591^{\circ} \mathrm{E}$ ], 6 Sept. 1987, J. R. I. Wood 5795 (E); Between Motithang and Phajoding, scattered on rather bare floor of open pine forest, by track, alt. $2600 \mathrm{~m}$ [27.486 ${ }^{\circ}$ N, $\left.89.598^{\circ} \mathrm{E}\right], 22$ Sept. 1987, J. R. I. Wood 5841 (E); Hill above Thimpu Hospital, damp slope in 


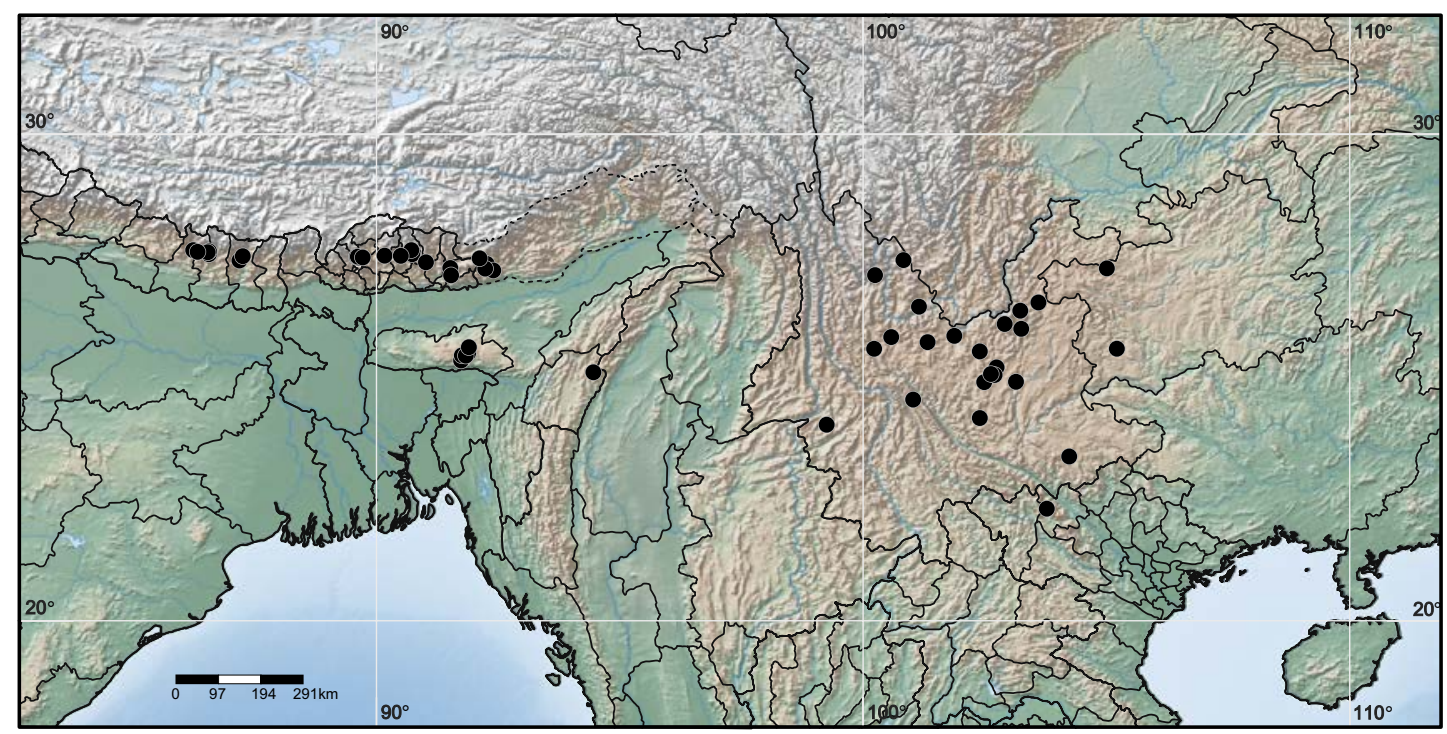

Fig. 1. General distribution of Calamagrostis abnormis (Hook. f.) Shukla.

open scrub, alt. $2400 \mathrm{~m}\left[27.465^{\circ} \mathrm{N}, 89.635^{\circ} \mathrm{E}\right], 6$ Aug. 1987, J. R. I. Wood 5668 (E); Road to microwave station above Motithang, Thimpu, wet cliff, by roadside, alt. $2580 \mathrm{~m}, 27.483^{\circ} \mathrm{N}, 89.617^{\circ} \mathrm{E}, 29$ Aug. 1998 , Noltie et al. 12 (E); Thimpu, hillside between microwave station and Motithang, relatively dense Pinus wallichiana forest, with Quercus semecarpifolia and other shrubs, alt. $2600 \mathrm{~m}, 27.483^{\circ} \mathrm{N}, 89.617^{\circ} \mathrm{E}, 29$ Aug. $1998, \mathrm{No}-$ ltie et al. 17 (E); Research Centre, Yosepang, weedy loam alt. $2550 \mathrm{~m}, 27.467^{\circ} \mathrm{N}, 89.717^{\circ} \mathrm{E}, 1$ Sept. 1998, Noltie et al. 47 (E). Trongsa Distr.: Tongsa, marshy hillside, alt. $2350 \mathrm{~m}\left[27.5^{\circ} \mathrm{N}, 90.5^{\circ} \mathrm{E}\right], 9$ July 1979 , A. J. C. Gierson \& D. G. Long 2628 (E). SamdruP JONGKHAR DisTR.: Deothang, Wamrong, roadside verge, alt. $2300 \mathrm{~m}, 27.1^{\circ} \mathrm{N}, 91.533^{\circ} \mathrm{E}, 12$ Sept. 1998 , Noltie et al. 180 (E).

CHINA. Guizhou. Xingren Co.: Xiashanxiang, grassland on slope, alt. $1340 \mathrm{~m}\left[25.59^{\circ} \mathrm{N}, 105.215^{\circ} \mathrm{E}\right]$, 23 June 1986, C. Z. Dang 13 (PE, 2 sheets); Qixingguan Distr.: Yangshan, slope, edge of wood, alt. $1450 \mathrm{~m}$ [27.242 $\left.{ }^{\circ} \mathrm{N}, 105.01^{\circ} \mathrm{E}\right], 17$ March 2003, H. Y. Ma 154 (KUN). YunNAN. Anning City: Wenquan Town, Yiweili, alt. $1880 \mathrm{~m}\left[24.904^{\circ} \mathrm{N}, 102.489^{\circ} \mathrm{E}\right], 17 \mathrm{Aug} .2006$, E. D. Liu 1826 (KUN); Wenquan, Mopanshan, alt. $2030 \mathrm{~m}$ $\left[24.904^{\circ} \mathrm{N}, 102.489^{\circ} \mathrm{E}\right], 16$ July 1965 , C. Y. Wu 347 (KUN); Anfengying, Yangtianchong, alt. ca $1900 \mathrm{~m}$, $\left.24.904^{\circ} \mathrm{N}, 102.489^{\circ} \mathrm{E}\right], 1$ July 2007, H. Peng et al. 48 (KUN). Binchuan Co.: Jizu Mt. [25.83N, $100.578^{\circ} \mathrm{E}$ ], alt. 2300 m, 22 Aug. 1963, NW-Yunnan-Jinshajiang Team 63-6639 (KUN). Dali City: Cangshan Mt., moist site, alt. $3000 \mathrm{~m}\left[25.59^{\circ} \mathrm{N}, 100.226^{\circ} \mathrm{E}\right], 1$ Oct. 2002 , H. Y. Ma 144 (KUN). Dayao Co.: Tanhuashan, alt. $2650 \mathrm{~m}\left[25.726^{\circ} \mathrm{N}, 101.325^{\circ} \mathrm{E}\right], 10$ Sept. 1960 , s.c. 60-058 (KUN); Tanhuashan $\left[25.726^{\circ} \mathrm{N}, 101.325^{\circ} \mathrm{E}\right]$, alt. $2500 \mathrm{~m}, 2$ Sept. 1963, NW-Yunnan-Jinshajiang Team 63-6803 (KUN). Dongchuan Distr.: Hongtudi Town, mountain behind Laotanfang, in thickets, alt. $3200 \mathrm{~m}$ [26.099 $\left.{ }^{\circ} \mathrm{N}, 102.91^{\circ} \mathrm{E}\right], 1$ Aug. 2008, H. Peng et al. 9212 (KUN). Eshan Co.: Chahexiang to Xiejia, sunny slope, alt. $1370-1890 \mathrm{~m}\left[24.166^{\circ} \mathrm{N}, 102.398^{\circ} \mathrm{E}\right], 11$ Oct. 1988 , Eshan Team 634 (KUN). Huaping Co.: on road from Panzhihua to Niglang, ca $30 \mathrm{~km} \mathrm{SW}$ of Huaping, $2100 \mathrm{~m}$, roadside in forest (walnut, Alnus, Pinus) with Galinsonga, Fagopyrum, Plantago, Conyza canadensis, Eragrostis, alt. $2100 \mathrm{~m}\left[26.455^{\circ} \mathrm{N}, 101.148^{\circ} \mathrm{E}\right], 16 \mathrm{Aug}$. 2010, Paszko GPS 604 (KRAM). Huize Co.: Liangwang Shan, $c a 20 \mathrm{~km}$ SE of Dongchuan, on new road to Zhoatong via Zhehai, scruby Pinus yunnanensis/ Alnus nepalensis forest, shrubs and grasses, around corn and tobacco fields, alt. $2080 \mathrm{~m}, 26^{\circ} \mathrm{N}, 103.25^{\circ} \mathrm{E}$, 14 Sept. 1997, Soreng et al. 5290 (KUN); Liangwang Shan, $c a 20 \mathrm{~km}$ ENE of Dongchuan, on new road to Zhoatong via Zhehai, near pass, alt. $2670 \mathrm{~m}, 26.372^{\circ} \mathrm{N}$, $103.23^{\circ}$ E, 14 Sept. 1997, Soreng et al. 5315 (KUN); Montagnes denudees, arides, calcaires a Tche-hai [Zhehaizhen], alt. $2990 \mathrm{~m}\left[26.541^{\circ} \mathrm{N}, 103.603^{\circ} \mathrm{E}\right], 1913$, E. E. Maire 1296 (E). Jingdong Yi Autonomous Co.: Taizhong, Xujiaba, Ecology Station of XTBG, marsh, alt. $2489 \mathrm{~m}\left[24.545^{\circ} \mathrm{N}, 101.029^{\circ} \mathrm{E}\right], 16$ Aug. 2006, T. Zhang et al. SCSB-A-000107 (KUN). Ninglang Yi 
Autonomous Co.: $\mathrm{N}$ of Ninglang, on the road from Niglang to Lugu $\mathrm{Hu}$ Lake, alt. $2610 \mathrm{~m}$, roadside on mountain slope, Pinus forest with Quercus, Alnus, Agrimonia, Plantago sp. and Fragaria, alt. $2100 \mathrm{~m}$, $27.412^{\circ} \mathrm{N}, 100.829^{\circ} \mathrm{E}, 17$ Aug. 2010, B. Paszko GPS 608 (KRAM). Panlong Distr.: S of Dashaocun village, alt. $2270 \mathrm{~m}$, roadside near mountain slope, $2270 \mathrm{~m}$, 25.207 ${ }^{\circ}$, $102.743^{\circ} \mathrm{E}, 14$ Aug. 2010, B. Paszko GPS 601 (KRAM); Kunming, Heilongtan, alt. ca $1900 \mathrm{~m}$ $\left[25.051^{\circ} \mathrm{N}, 102.702^{\circ} \mathrm{E}\right], 15$ July 1954 , Kunming Institute of Botany 50507 (KUN), 16 June 1941, F. T. Wang 2053 (KUN), 1 July 1941, F. T. Wang 2193 (KUN), 19 July 1954, s.c. 5052 (KUN), 24 Aug. 2001, H. Y. Ma 1 (KUN), 15 June 1941, F. T. Wang 2127 (KUN), 17 Oct. 2010, G. F. Li 657 (KUN). Wenshan City: Laojunshan, alt. $2000 \mathrm{~m}, 23.376^{\circ} \mathrm{N}, 104.235^{\circ} \mathrm{E}$ ], 26 Sept. $1992, Y . M$. Shui 335 (KUN). Wuding Co.: Guanpo, Shilouti Reservoir, among secondary bushes $\left[25.536^{\circ} \mathrm{N}, 102.398^{\circ} \mathrm{E}\right]$, alt. 2590 m, 14 Apr. 2003, H. Li et al. 1379 (KUN). Xishan Distr.: Kunming, Western Hills, alt. ca $2100 \mathrm{~m}$ [25.051 $\left.{ }^{\circ} \mathrm{N}, 102.702^{\circ} \mathrm{E}\right], 4$ Aug. 2007, Y. C. Liu \& N. Hu 127 (KUN). Yiliang Co.: Xiaoguanglihuazhuang, under forest on slope $\left[24.911^{\circ} \mathrm{N}, 103.142^{\circ} \mathrm{E}\right], 26$ Aug. 1975 , B. Y. Qiu 60660 (KUN). Yongde Co.: Wumulong, Ganhedapo, alt. $2400 \mathrm{~m}\left[24.029^{\circ} \mathrm{N}, 99.251^{\circ} \mathrm{E}\right], 18$ June 2003, E. D. Liu \& D. C. Yang 51 (KUN); Wumutuo, alt. $2050 \mathrm{~m}\left[24.029^{\circ} \mathrm{N}, 99.251^{\circ} \mathrm{E}\right], 18$ July $2002, E$. D. Liu 5228 (KUN).

india. Arunachal Pradesh. West Kameng Distr. / CHINA. Xizang. Cona Co. (disputed area): Dirang Dzong, scattered in open pine-oak woods, alt. $1525-1830 \mathrm{~m}\left[27.333^{\circ} \mathrm{N}, 92.267^{\circ} \mathrm{E}\right], 26$ July 1938 , F. Kingdon-Ward 14001 (BM, E); Rupa, alt. 1830$2130 \mathrm{~m}$, damp places in climax pine-oak $\left[27.2^{\circ} \mathrm{N}\right.$, $\left.92.4^{\circ} \mathrm{E}\right], 13$ July 1938, F. Kingdon-Ward 13905 (BM, E); Phutang [Phudung village], alt. ca $2130 \mathrm{~m}\left[27.237^{\circ} \mathrm{N}\right.$, 92.234 E], 8 July 1938, F. Kingdon-Ward 13880 (BM); Senge Dzong [in Sela Range], climax pine-oak, alt. 2130-2440 m $\left[27.448^{\circ} \mathrm{N}, 92.12^{\circ} \mathrm{E}\right], 28$ Aug. 1938, F. Kingdon-Ward $14172 A$ \& 14174 (BM). MANIPUR: Sirhoi Kashong [Shirui Kashong Peak], alt. 2285$2590 \mathrm{~m}\left[25.106^{\circ} \mathrm{N}, 94.458^{\circ} \mathrm{E}\right], 27$ July 1948 , KingdonWard $17882(\mathrm{~K})$. Meghalaya: East Khasi Hills Distr.: Khasi Hills, "Agrostis 12", Nonkreem [Nongkrem village] $\left[25.5^{\circ} \mathrm{N}, 91.883^{\circ} \mathrm{E}\right]$, J. D. Hooker \& T. Thompson s.n. (K, p.p.); Khasi Hills, Agrostis 12, Surureem [Serrarim, Sohra rin, Sohrarim] [25.349 $\left.\mathrm{N}, 91.739^{\circ} \mathrm{E}\right]$, 26 June 1850, J. D. Hooker \& T. Thompson 1232 (K); Khasi Hills, Mawphlang, grassland, alt. $1830 \mathrm{~m}$ [25.453 $\left.{ }^{\circ} \mathrm{N}, 91.754^{\circ} \mathrm{E}\right], 7$ Aug. 1954, T. R. Chand 7929 (W, 2 sheets; US); Laitlynkot [Laitlyngkot], Khasi Hills, alt. $1830 \mathrm{~m}$, meadow $\left[25.448^{\circ} \mathrm{N}, 91.835^{\circ} \mathrm{E}\right], 12$ July
1949, W. N. Koelz 23175 (US); Khasi Hills, Shillong, alt. $1530 \mathrm{~m}\left[25.622^{\circ} \mathrm{N}, 91.899^{\circ} \mathrm{E}\right], 1$ Aug. 1885, C. B. Clarke $44382 A$ (LE). Unlocated Distr.: Khasi Hills, Agrostis 12, alt. 1525-1830 m, J. D. Hooker \& T. Thompson s.n. (BM; E, 2 sheets; K; L, 2 sheets; LE p.p., 3 sheets; P, 4 sheets; $\mathrm{W}, 4$ sheets).

NEPAL. Dolakha Distr.: Jiri $(1860 \mathrm{~m})-$ Bkal (2020 m) - Ratmate (2270 m) - Maligaon (2200 m) Shivalaya $(1800 \mathrm{~m})$, alt. $2100 \mathrm{~m}$, on open moist place, $27.607^{\circ} \mathrm{N}, 86.257^{\circ} \mathrm{E}, 4$ July $1985, \mathrm{H}$. Ohba et al. 60010 (TI); Jiri (1860 m) - Bkal (2020 m) - Ratmate (2270 m) - Maligaon (2200 m) - Shivalaya (1800 m), alt. $2200 \mathrm{~m}$, on moist ground along stream, $27.617^{\circ} \mathrm{N}, 86.233^{\circ} \mathrm{E}$, 4 July 1985, H. Ohba et al. 60023 (TI). RAMECHHAP DisTR.: Shivalaya (1800 m) - Khasrubus (2400 m) Deolari $(2700 \mathrm{~m})$ - Bhadar $(2300 \mathrm{~m})$, alt. $2550 \mathrm{~m}$, $27.582^{\circ} \mathrm{N}, 86.323^{\circ} \mathrm{E}, 17$ Aug. 1985, H. Ohba et al. 61357 (TI); Shivalaya (1800 m) - Khasrubus (2400 m) - Deorali $(2700 \mathrm{~m})$, alt. $2100 \mathrm{~m}$, along trail, on open slope, $27.582^{\circ} \mathrm{N}, 86.323^{\circ} \mathrm{E}, 5$ July $1985, H$. Ohba et al. 60065 (TI). SANKHUWASABHA DisTR.: Manebanjang (1130 m) Danda Pangma (1160 m) Sekaha (1460 m) Chipe gaun $(1570 \mathrm{~m})$ - Bhotenbas (1830 m) - Gogane (1910 m) - Chichila (1910 m), on edge of Castanopsis forests, alt. $1800-1900 \mathrm{~m}, 27.404^{\circ} \mathrm{N}, 87.184^{\circ} \mathrm{E}, 28$ July 1990 , N. Minaki et al. 90-20199 (TI); Makalu-Barum National Park, Bhotebes $(1810 \mathrm{~m})-$ Cogane $(1880 \mathrm{~m})-$ Chichila (1840m) - Kaptane (2000m), alt. 1880 m, dotted on shady bank, $27.493^{\circ} \mathrm{N}, 87.252^{\circ} \mathrm{E}, 17$ Aug. 1997, S. Noshiro et al. 97-70142 (BM). SolukhumBu Distr.: Beni $(2350 \mathrm{~m})$ - Lekhani $(2700 \mathrm{~m})$ - Tambakhani (2800 m) - Loding Khola (2350 m) - Loding (2600 m), alt. $2400 \mathrm{~m}$, on open slope along trail, alt. $2400 \mathrm{~m}$, $27.534^{\circ} \mathrm{N}, 86.556^{\circ} \mathrm{E}, 4$ Sept. 1985, H. Ohba et al. 62069 (TI); Mopung (2800 m) - Thosa Kharka (3670 m), alt. $3000 \mathrm{~m}$, on open slope, $27.594^{\circ} \mathrm{N}, 86.558^{\circ} \mathrm{E}, 21 \mathrm{Aug}$. 1985, H. Ohba et al. Wu 61546 (TI); Taktor (3000 m) - Junbesi (2650 m) - Mopung (2800 m), alt. $2800 \mathrm{~m}$, on open slope along trail, $27.573^{\circ} \mathrm{N}, 86.532^{\circ} \mathrm{E}, 20 \mathrm{Aug}$. 1985, H. Ohba et al. 61502 (TI).

VIETNAM. LaO Kay (LÀo CAI). Sa Pa Distr.: Chapu [Chapa, Sa Pá, Sa Pa, Sapa], Massif du Fan Tsi Pan [Phan Xi Păng, Fansipan], alt. 2900 m [22.303 N, 103.775 E), Aug. 1942, Petelot 7988 (P03252805, $\mathrm{P} 02651396)$.

2. Calamagrostis extremiorientalis (Tzvel.) Prob. Sosud. Rast. Sovetsk. Dal'nego Vostoka 1: 200. 1985. BAsIOnYm: Calamagrostis epigeios (L.) Roth subsp. extremiorientalis Tzvel., Novosti Sist. Vyssh. Rast. 2: 40. 1965. C. epigeios (L.) Roth var. extremiorientalis (Tzvel.) Kitag., Neo-Lineam. Fl. Manshur.: 74. 1979. 


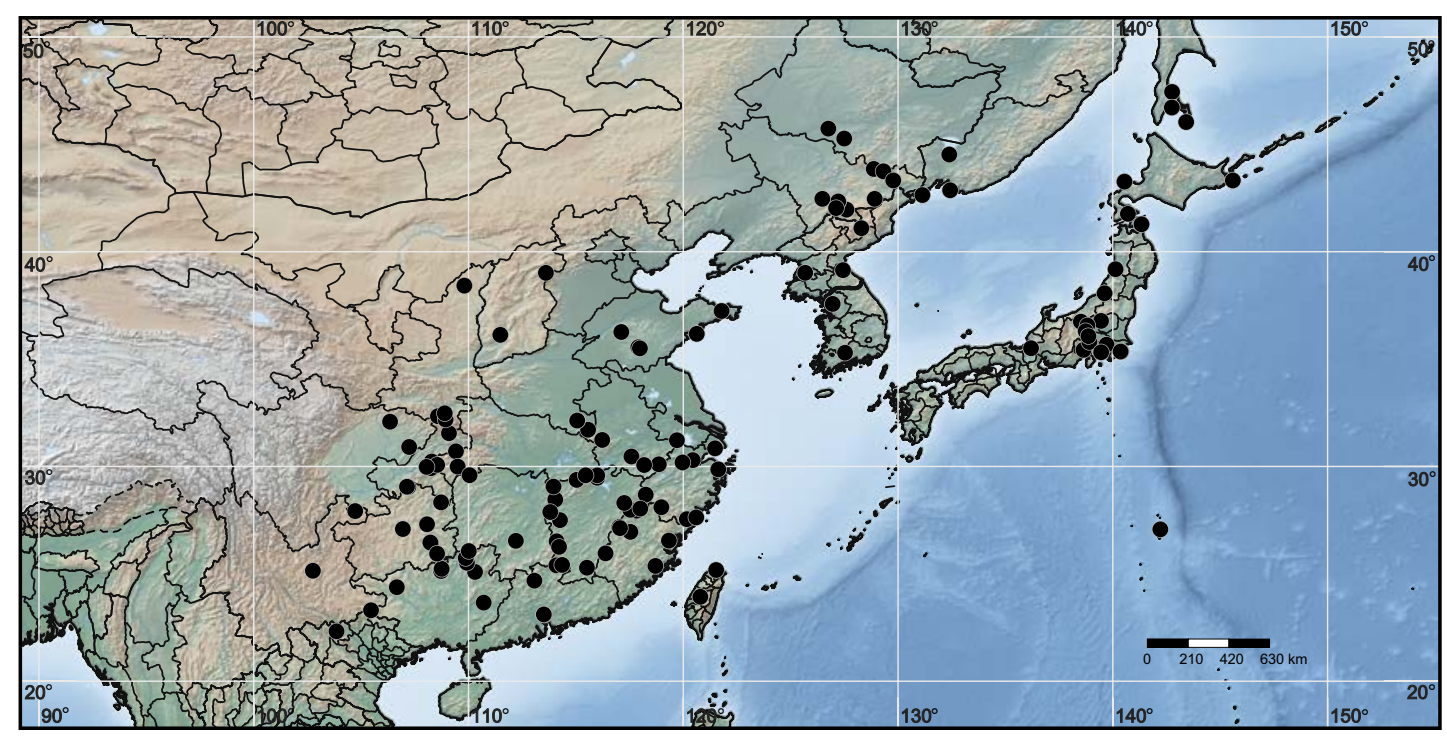

Fig. 2. General distribution of Calamagrostis extremiorientalis (Tzvel.) Prob.

TyPe: RUSSIA. [SAKHALIN OBLAST]. [Korsakovsky Distr.]: Yuzhn. Sakhalin (S Sakhalin), Anivskij zaliv [Aniva Bay], g. Aniva (Mt. Aniva), on sand dunes at seashore $\left[46.036^{\circ} \mathrm{N}, 143.416^{\circ} \mathrm{E}\right], 12$ Aug. 1948, M. Popov s.n. (HOLOTYPE, LE!).

Calamagrostis kengii T. F. Wang, Acta Phytotax. Sin. 10: 312. 1965. Type: CHINA. HeILONGJIANG. [Ning'an City]: Shanchi (Shangzhi), Maorshan (Mao'ershan), the forest of NEFI [ $\left.45.268^{\circ} \mathrm{N}, 127.495^{\circ} \mathrm{E}\right], 13$ July 1961 , Y. B. Chang (Y. B. Zhang) \& G. J. Yin (G. Z. Ren) 3660 (HOLOTYPe, NEFI!). Synonymized by Paszko \& Ma (2011).

Calamagrostis epigeios (L.) Roth var. sylvatica T. F. Wang, Acta Phytotax. Sin. 10: 309. 1965. TYPE: CHINA. JiLIN. [Changbai Korean Autonomous Co.]: Linkiang (Linjiang), S edge of Mt. Changbaishan $\left[42.032^{\circ} \mathrm{N}\right.$, 127.116 ${ }^{\circ}$ E], 20 Aug. 1957, Y. L. Chou (Y. L. Zhou) 2442 (HOLOTYPe, NEFI!). Synonymized by Paszko \& Ma (2011).

Calamagrostis elatior auct. non (Griseb.) A. Camus: Camus, Bull. Soc. Bot. France 75: 555. 1928; Schmid, Agron. Trop. (Nogent-sur-Marne) 13: 484. 1958; Khoi \& Đo, Checkl. Pl. Spec. Vietnam 3: 782. 2005.

Calamagrostis epigeios auct. non (L.) Roth (p.p.): Lu, Fl. Reipubl. Popularis Sin. 9(3): 229. 1987; Lu \& Phillips, Fl. China 22: 360. 2006.

A reliable description of $C$. extremiorientalis was provided by Paszko and Ma (2011).
Calamagrostis elatior was recorded by Camus (1928) and subsequently by Schmid (1958) from Tonkin Province in Vietnam based on Petelot's collection no. 5080, housed at P. During the $18^{\text {th }}$ and $19^{\text {th }}$ centuries, westerners commonly used the name Tonkin (from Đông Kinh) to refer to northwestern Vietnam. The species was recorded from $\mathrm{Sa} \mathrm{Pa}$ [the French called it Chapa], the tourist capital of northwestern, mountainous Vietnam, situated in the Tonkin Alps (Camus 1928; Schmid 1958). Morphological examination of this collection showed that these specimens belong to $C$. extremiorientalis, a species not previously recorded from Vietnam.

Habitat AND PHENOLOGy. Perennial grass with long rhizomes. It occupies roadsides, uncultivated fields, grassy mountain slopes, riversides, open places in forests, sand dunes and swampy meadow at the seashore. 0-2000 m. Flowering June-August.

General distribution. China (Anhui, Chongqing, Fujian, Guangdong, Guangxi, Guizhou, Heilongjiang, Henan, Hubei, Hunan, Jiangsu, Jiangxi, Jilin, Liaoning, S Shaanxi, Shandong, Shanghai, Shanxi, E Sichuan, Taiwan, E Yunnan, Zhejiang), Japan (Hokkaido, Honshu, Ogasawara Islands), North Korea (Kangwon, Pyongyang, 
Ryanggang), Russia (Amur Oblast, Khabarovsk Krai, Primorsky Krai, S Sakhalin Oblast), South Korea (Jeolanam, Seoul), northwestern Vietnam (Lao Kay) (Fig. 2).

Notes. Calamagrostis epigeios subsp. extremiorientalis was described by Tzvelev (1965: 40) on the basis of Popov's collection from the southern part of Sakhalin Island (Russian Far East). Tzvelev $(1965,1976)$ recorded it from the southern part of the Russian Far East (S Khabarovsk Krai, Primorsky Krai, S Sakhalin), Japan, the Korean Peninsula and northeastern China. Probatova (1985) provided a dot distribution map of $C$. extremiorientalis in the Russian Far East. She recorded C. extremiorientalis in Primorsky Krai and in the southern parts of the following subdivisions: Amur Oblast, Khabarovsk Krai, Sakhalin Island, Kuril Islands (Probatova 1985). The taxonomy of the $C$. epigeios complex in China was clarified by Paszko and Ma (2011) with the recognition of three species in China: C. epigeios, C. extremiorientalis and C. macrolepis. Paszko and Ma (2011) wrongly cited localities from Xuanen County within Hebei Province; Xuanen is a county of southwestern Hubei Province, China. As such, confirmed records of $C$. extremiorientalis from Hebei are lacking.

We here provide the first record of $C$. extremiorientalis from northwestern Vietnam and Japan's remote Bonin Islands (also known as the Ogasawara Islands). Its occurrence in Vietnam represents the southern limit of its known geographical range. No species of Calamagrostis is recorded in the Oceanic Islands Plant Specimen Database (Anonymous 2017), which was established to better understand the flora of the islands in Japan (Ohi-Toma et al. 2015).

SPECIMENS EXAMINED: CHINA. ANHUI. Guichi Distr.: Meijie Town, alt. $200 \mathrm{~m}\left[30.46^{\circ} \mathrm{N}, 117.59^{\circ} \mathrm{E}\right], 1 \mathrm{July}$ 1959, s.c. 7036 (PE). Huangshan Distr.: Mt. Huangshan (Mt. Huang, Mt. Yellow), Chuishihlin to Tangkow $\left[30.07^{\circ} \mathrm{N}, 118.18^{\circ} \mathrm{E}\right], 6$ Aug. $1935, T . N$. Liou \& P. C. Tsoong 2177 (PE). Huoshan Co.: Dabeiping $\left[31.23^{\circ} \mathrm{N}\right.$, $\left.116.23^{\circ} \mathrm{E}\right], 30$ June 1959, s.c. 40487 (PE). Jinzhai Co.: Jingangtai Nature Protection Area $\left[31.71^{\circ} \mathrm{N}, 115.57^{\circ} \mathrm{E}\right]$, 12 July 1959, s.c. 60997 (PE). Jixi Co.: Qingliangfeng National Nature Reserve, mountain top, grassland, alt. $1780 \mathrm{~m}\left[30.1^{\circ} \mathrm{N}, 118.86^{\circ} \mathrm{E}\right], 23$ Aug. $1973, X$. Zhou
\& G. Pan 580 (PE). ChongQIng. Dazu Distr.: Qinghe Town, Wanliping, alt. $1140 \mathrm{~m}\left[30.91^{\circ} \mathrm{N}, 107.24^{\circ} \mathrm{E}\right]$, 20 July 1963, Z. R. Zhang 76 (CDBI). Fengjie Co.: Xinhe Township, Xinjie, alt. $1100 \mathrm{~m}\left[30.7^{\circ} \mathrm{N}, 109.42^{\circ} \mathrm{E}\right]$, 29 June 1958, H. F. Zhou 26484 (KUN). Nanchuan Distr.: Yezhudang, Yangyuping, Mt. Jinfoshan, alt. $1450-1750 \mathrm{~m}\left[29.05^{\circ} \mathrm{N}, 107.2^{\circ} \mathrm{E}\right], 27$ July 1978 , Phytogeography Group 736 (CDBI); Mt. Jinfo, below the Daheba, alt. $1100 \mathrm{~m}\left[29.06^{\circ} \mathrm{N}, 107.13^{\circ} \mathrm{E}\right], 6$ July 1957 , G. F. Li 62550 (KUN); Changlingang, Xinchangping, Mt. Jinfo, alt. $1700 \mathrm{~m}\left[29.06^{\circ} \mathrm{N}, 107.13^{\circ} \mathrm{E}\right], 12$ July 1957, G. F. Li 62677 (KUN). Shizhu Tujia Autonomous Co.: Chizitang, Qiyueshan Forest Farm, alt. 1710 m $\left[30^{\circ} \mathrm{N}, 108.12^{\circ} \mathrm{E}\right], 11$ July 1978, Y. Chen 2164 (CDBI); Yuelaizhen, Mt. Fangdoushan, Qingcaopo grassy slope, alt. $900-1260 \mathrm{~m}\left[30.23^{\circ} \mathrm{N}, 108.29^{\circ} \mathrm{E}\right], 17$ July 1988 , Y. L. Cao \& F. D. Fu 838 (CDBI); Sanhui Township, alt. $1000 \mathrm{~m}\left[29.92^{\circ} \mathrm{N}, 108.25^{\circ} \mathrm{E}\right], 28$ June 1978, W. H. Wang 1734 (CDBI); Chizitang, Qiyueshan Forest Farm, alt. $1600 \mathrm{~m}\left[30.07^{\circ} \mathrm{N}, 108.54^{\circ} \mathrm{E}\right], 21$ July $1978, Y$. Chen 2877 (CDBI). Wuxi Co.: Hongchiba, alt. $1870 \mathrm{~m}\left[31.55^{\circ} \mathrm{N}\right.$, $\left.109.09^{\circ} \mathrm{E}\right], 1$ Aug. 1989, Y. H. Liu et al. 89084 (PE). Youyang Tujia and Miao Autonomous Co.: Qinghua Forest Farm, alt. $1200 \mathrm{~m}\left[29.98^{\circ} \mathrm{N}, 108.05^{\circ} \mathrm{E}\right], 17 \mathrm{Aug}$. 1989, Z. C. Zhao \& J. S. Ma 3027 (CDBI). Fujian. Changting Co.: In monte Tienhwa-schan ad occ. Dingdschou (Tingchow), in meadow [ $25.954^{\circ} \mathrm{N}, 116.381^{\circ} \mathrm{E}$ ], June/July 1921, T.H. Wang 423 (W). Shunchang Co.: Jiatou Village, alt. $500 \mathrm{~m}\left[26.95^{\circ} \mathrm{N}, 117.54^{\circ} \mathrm{E}\right], 17$ July 1986, M. S. Li \& Z. Y. Li 5699 (PE). Wuyishan City: Qianshan, Xingcun Town, Wushi, alt. $550 \mathrm{~m}\left[27.94^{\circ} \mathrm{N}\right.$, $117.83^{\circ} \mathrm{E}$ ], 27 June 1998, M. Susuki et al. 273 (PE); Xikeng Village, Qianshan, alt. $1000 \mathrm{~m}\left[27.98^{\circ} \mathrm{N}\right.$, $117.53^{\circ} \mathrm{E}$ ], 17 Aug. 1958, s.c. 4203 (KUN). Xianyou Co.: $\left[25.37^{\circ} \mathrm{N}, 118.70^{\circ} \mathrm{E}\right], 30$ June 1931, Y. Lin 429 (PE). Yongding Distr.: [26.55N, $\left.119.33^{\circ} \mathrm{E}\right]$, July 1943, R. Lin 3030 (PE). GuANGdONG. Huangpu Distr.: [Nangang Town], Iu Village, Lung Tau Shan [Longtou Hill] [23.108 $\left.{ }^{\circ} \mathrm{N}, 113.506^{\circ} \mathrm{E}\right], 19$ July 1924, To \& Tsang 13019 (PE, S). Ruyuan Yao Autonomous Co.: Luoyang Village, Banpengshan $\left[24.68^{\circ} \mathrm{N}, 113.07^{\circ} \mathrm{E}\right], 3$ July $1957, Y$. G. Liu 604 (PE). GuangXI. Jingxi City: Damiaoshan, Sanfangqu, Chitong, Jiuwanshan, Mt. behind the Yuya, alt. $1100-1250 \mathrm{~m}\left[25.13^{\circ} \mathrm{N}, 108.73^{\circ} \mathrm{E}\right], 26$ June $1958, S . Q$. Chen 14699 (KUN); Damiaoshan, Ludongxiang, Gema, Jiuwan Dashan, alt. $650 \mathrm{~m}\left[25.23^{\circ} \mathrm{N}, 108.75^{\circ} \mathrm{E}\right], 12$ June 1957, D. Z. Chen 720 (KUN). Lingui Distr.: around Mt. Yan $\left[25.09^{\circ} \mathrm{N}, 110.30^{\circ} \mathrm{E}\right]$, s.d., G. B. Xu $10320(\mathrm{KUN})$; Huangsha Township, Diaopeng $\left[25.51^{\circ} \mathrm{N}, 109.93^{\circ} \mathrm{E}\right]$, 7 June 1958, Z. Z. Chen 50910 (KUN, PE). Lingyun Co.: Loh Hoh Tsuen, Ling Yun Hsien, hillside, alt. $1150 \mathrm{~m}$ [24.377 ${ }^{\circ}$, $\left.106.662^{\circ} \mathrm{E}\right], 20$ July 1933, A. N. Steward 
\& H.C. Cheo 716 (W). Longsheng Various Nationalities Autonomous Co.: Sanmen, Dadi, top of Mt. Hongya, alt. $1540 \mathrm{~m}\left[25.73^{\circ} \mathrm{N}, 109.87^{\circ} \mathrm{E}\right], 13$ Aug. 1957, S. L. Wang 3667 (KUN); Dadi Township, Taiping Village, alt. $1000 \mathrm{~m}\left[26.06^{\circ} \mathrm{N}, 110.01^{\circ} \mathrm{E}\right], 5$ July 1955 , The Forest Plant Group of Guangdong, Guangxi and Fujian 734 (PE). Teng Co.: Taiping Town, Lion Hill, $25 \mathrm{Li}$ [ca $12.5 \mathrm{~km}] \mathrm{N}$ of Luchon, alt. $300 \mathrm{~m}\left[23.66^{\circ} \mathrm{N}, 110.71^{\circ} \mathrm{E}\right]$, 28 May 1920, R. C. Ching 5429 (PE). GuIzHou. Huangping Co.: Shibing, at the foot of Mt. Foding $\left[27.31^{\circ} \mathrm{N}\right.$, $108.07^{\circ} \mathrm{E}$ ], 21 July 1959, Qiannan Team 2560 (KUN). Kaiyang Co.: In pratis dumosis inter urbem Kweiyang [Kaiyang] et vic. Guangyinschan copiose, alt. 1100$1300 \mathrm{~m}\left[27.087^{\circ} \mathrm{N}, 106.943^{\circ} \mathrm{E}\right], 6$ July 1917, H. HandelMazzettii 2068 (W). Leishan Co.: between Shuishe and Qiaotou, Jianbanliang, alt. $900 \mathrm{~m}\left[26.42^{\circ} \mathrm{N}, 108.26^{\circ} \mathrm{E}\right]$, 1 July 1965, Z. P. Jian et al. 50971 (KUN); SW slope of Mt. Leigong Ping, alt. $2000 \mathrm{~m}\left[26.46^{\circ} \mathrm{N}, 108.22^{\circ} \mathrm{E}\right]$, 7 July 1959, Z. P. Jian et al. 51224 (KUN). Rongjiang Co.: Jiamin Township, Yanjiang [25.94 $\left.\mathrm{N}, 108.54^{\circ} \mathrm{E}\right]$, 30 July 1959, Qiannan Team 3024 (KUN). Songtao Miao Autonomous Co.: Ganlong, Mt. Zhengjiashan, alt. $780 \mathrm{~m}\left[28.33^{\circ} \mathrm{N}, 108.72^{\circ} \mathrm{E}\right], 6$ July 1959, T. P. Zhu \& Z. F. Liu 1279 (KUN). Henan. Huangchuan Co.: Buyang Valley [ $\left.32.14^{\circ} \mathrm{N}, 115.06^{\circ} \mathrm{E}\right]$, July 1959 , General Survey Group 29097 (PE); Mt. Buyananshan [32.18 N, $\left.115.06^{\circ} \mathrm{E}\right]$, July 1959, General Survey Group 29113 \& 29116 (PE).

Heilonguiang. Ning'an City: W bank of Jingpo Lake, alt. $350 \mathrm{~m}\left[43.85^{\circ} \mathrm{N}, 128.87^{\circ} \mathrm{E}\right], 6$ Sept. 1981 , G. S. Zhou et al. 22 (PE). Xiangfang Distr.: Vicinity of Harbin: Siaolon, Wiese bei Caocun [meadow near Caocun] [ $\left.45.731^{\circ} \mathrm{N}, 126.753^{\circ} \mathrm{E}\right], 11$ July 1926 , Jettmar s.n. (W). Huber. Xuanen Co.: [illegible], alt. $1400 \mathrm{~m}$, 5 July 1975, H. J. Li 3551 (KUN); [without precise locality], alt. $1000 \mathrm{~m}\left[29.993^{\circ} \mathrm{N}, 109.495^{\circ} \mathrm{N}\right]$, s.d., H. J. Li 3872 (PE); s.d., H. J. Li 3868 (PE); 20 July 1958, H. J. Li 5060 (PE). HunAN. Changning City: Yang-Shan, Changning Hsien, grassy slope, alt. $680 \mathrm{~m}\left[26.536^{\circ} \mathrm{N}\right.$, $\left.112.205^{\circ} \mathrm{E}\right], 10 \mathrm{July} 1935$, C. S. Fan \& Y. Y. Li 220 (W). Sangzhi Co.: Shadiping, Lujiabao, alt. $1000 \mathrm{~m}\left[29.59^{\circ} \mathrm{N}\right.$, $\left.110.05^{\circ} \mathrm{E}\right]$, 1 July 1958, L. H. Liu 9235 (KUN). JiANGSU. Yixing City: Mt. Longchi [ $\left.31.22^{\circ} \mathrm{N}, 119.70^{\circ} \mathrm{E}\right], 25 \mathrm{June}$ 1962, S. H. Mao et al. 76 (KUN). JiAngXI. Anfu Co.: Wugong shan, alt. $320 \mathrm{~m}\left[27.50^{\circ} \mathrm{N}, 114.26^{\circ} \mathrm{E}\right], 2 \mathrm{Aug}$. 1963, J. S. Yue 2840 (KUN). Anyuan Distr.: Aibei, Dujiang Township $\left[25.29^{\circ} \mathrm{N}, 115.51^{\circ} \mathrm{E}\right]$, s.d., s.c. 2848 (KUN); Dujiang Township [25.29 ${ }^{\circ} \mathrm{N}, 115.51^{\circ} \mathrm{E}$ ], 20 June 1958, s.c. 2826 (KUN). Dayu Co.: Neiliang Township, Hailuogang $\left[25.4^{\circ} \mathrm{N}, 114.09^{\circ} \mathrm{E}\right], 19$ June 1962, J. S. Yue et al. 1422 (KUN); Mt. Bayun, alt. $800 \mathrm{~m}\left[25.42^{\circ} \mathrm{N}, 114.36^{\circ} \mathrm{E}\right], 10$ July $1962, J$. S. Yue et al. 1582 (KUN). Guixi City: Lengshui Township, alt. $320 \mathrm{~m}\left[28.3^{\circ} \mathrm{N}, 117.25^{\circ} \mathrm{E}\right], 20$ July $1958, M$. X. Nie \& S. S. Lai 3645 (KUN, PE). Jinggangshan City: Mt. Jingzhu, alt. $1300 \mathrm{~m}\left[26.51^{\circ} \mathrm{N}, 114.1^{\circ} \mathrm{E}\right], 30$ June 1965 , S. S. Lai et al. 4326 (KUN). Lichuan Co.: Deshengguan [27.18 $\left.{ }^{\circ} \mathrm{N}, 117.02^{\circ} \mathrm{E}\right], 16$ June $1958, M . X$. Nie \& S. S. Lai 2954 (KUN). Lichuan Co.: Honglin, Dayuan Forest [27.13으, $\left.117.05^{\circ} \mathrm{E}\right], 7$ June $1958, M . X$. Nie \& S. S. Lai 2848 (PE). Lushan City: Shahuaya, Mt. Lushan, alt. $1000 \mathrm{~m}\left[29.58^{\circ} \mathrm{N}, 115.99^{\circ} \mathrm{E}\right], 4$ Sept. $1963, M . X$. Nie 7797 (KUN); (Formerly Xingzi Co.), Xiufeng Temple, Hecun Road, alt. $200 \mathrm{~m}\left[29.47^{\circ} \mathrm{N}, 115.99^{\circ} \mathrm{E}\right], 24$ June 1963, M. X. Nie 7492 (KUN). Ruichang City: Qingshan, alt. $900 \mathrm{~m}\left[29.59^{\circ} \mathrm{N}, 115.45^{\circ} \mathrm{E}\right], 15$ Aug. $1995, C . M$. Tan 95560 (KRAM). Suichuan Co.: Dafen, Dabaling, alt. $1500 \mathrm{~m}\left[26.26^{\circ} \mathrm{N}, 114.22^{\circ} \mathrm{E}\right], 28$ Sept. $1963, J . S$. Yue 4378 (KUN). Shangli Co.: Circa carbonis minas Pinghsiang, alt. $600 \mathrm{~m}\left[27.879^{\circ} \mathrm{N}, 113.809^{\circ} \mathrm{E}\right], 1920$, T.H. Wang 22 (W). Shangrao Co.: Mt. Wufu, alt. $1100 \mathrm{~m}$ [28.04 N, $\left.117.998^{\circ} \mathrm{E}\right], 16$ Sept. 1958, M. X. Nie \& S. S. Lai 5111 (KUN). Tonggu Co.: Mt. Dawei $\left[28.46^{\circ} \mathrm{N}\right.$, $\left.114.04^{\circ} \mathrm{E}\right], 27$ Sept. 1963, S. S. Lai 3806 (KUN). Wuning Co.: Yishan Township, Changbang, alt. $300 \mathrm{~m}\left[29.37^{\circ} \mathrm{N}\right.$, $\left.115.04^{\circ} \mathrm{E}\right], 16$ June 1963, S. S. Lai 2632 (KUN). Xiushui Co.: Wenquan Township, Mt. Huanglong, alt. $1500 \mathrm{~m}$ [29.06 N, $\left.113.96^{\circ} \mathrm{E}\right], 24$ July 1963, S. S. Lai et al. 2862 (KUN). Yushan Co.: Mt. Hengyu [28.69 $\left.{ }^{\circ} \mathrm{N}, 118.25^{\circ} \mathrm{E}\right]$, 9 Nov. 1958, M. X. Nie \& S. S. Lai 6284 (KUN). JiLIN: Fusong Co.: Mt. Changbai, hills behind Manjiang, alt. $900 \mathrm{~m}\left[41.96^{\circ} \mathrm{N}, 127.6^{\circ} \mathrm{E}\right], 24$ July 1957, J. J. Qian et al. 281 (KUN). Helong City: Huancheng Township, Mt. Qing [42.46 $\left.{ }^{\circ} \mathrm{N}, 128.91^{\circ} \mathrm{E}\right], 23$ Aug. 1958, C. $S$. Wang et al. 2473 (KUN). Jingyu Co.: between Yushuchuan to Yusongjian, $480 \mathrm{~m}\left[42.34^{\circ} \mathrm{N}, 127.22^{\circ} \mathrm{E}\right]$, 7 Aug. 1950, D. G. Wang 1231 (KUN); Jiuliban Dianzi, alt. $590 \mathrm{~m}\left[42.46^{\circ} \mathrm{N}, 126.48^{\circ} \mathrm{E}\right], 28$ June $1958, S$. E. Liu et al. 1135 (KUN). Wangqing Co.: Chunyang Township, Gaoli Village, Jinkuangdong (Gold Mining Cave) [43.74으, $\left.129.32^{\circ} \mathrm{E}\right], 5$ Aug. 1959, P. Y. Fu 915 (PE); [s.l., $\left.43.32^{\circ} \mathrm{N}, 129.78^{\circ} \mathrm{E}\right], 20$ July $1958, C$. S. Wang et al. 1417 (KUN). LiAONING. Unlocated: Nianzhuang, s.d., Y. L. Zhang \& S. Q. Li 1051 (KUN). ShaAnXI. Langao Co.: Taohe Town, Zeping, alt. $1850 \mathrm{~m}\left[32.18^{\circ} \mathrm{N}\right.$, $\left.108.93^{\circ} \mathrm{E}\right], 25$ July 1959 , P. Y. Li 7762 (KUN); Near Shanyu, alt. $1800 \mathrm{~m}\left[32.48^{\circ} \mathrm{N}, 108.89^{\circ} \mathrm{E}\right], 20$ July 1959 , P.Y. Li 8218 (KUN). Yuyang Distr.: China bor., Ordos: Inter Yulin et Hungtao [38.42 ${ }^{\circ} \mathrm{N}, 109.807^{\circ} \mathrm{E}$ ], 26 July 1922, E. Licent s.n. (W). Ziyang Co.: Shuanghe Town, Qiangyazi, alt. $1750 \mathrm{~m}\left[32.34^{\circ} \mathrm{N}, 108.58^{\circ} \mathrm{E}\right], 16 \mathrm{Aug}$. 1959, P. Y. Li 7208 (KUN). Shandong. Fei Co.: Meng Shan $\left[35.501^{\circ} \mathrm{N}, 117.981^{\circ} \mathrm{E}\right], 21$ July $1936, T . Y$. Cheo \& L. Yen $130(\mathrm{~W})$. Pingyi Co.: Mt. Mengshan $\left[35.55^{\circ} \mathrm{N}\right.$, 
$117.91^{\circ} \mathrm{E}$ ], 25 July 1959, T. Y. Zhou et al. 6123 (PE). Laoshan Distr.: Mt. Laoshan [36.18 $\left.{ }^{\circ} \mathrm{N}, 120.63^{\circ} \mathrm{E}\right]$, 15 July 1930, C. Y. Chiao 2802 (PE). Taishan Distr.: Tai'an, top of Mt. Taishan $\left[36.26^{\circ} \mathrm{N} 4,117.11^{\circ} \mathrm{E}\right]$, 19 July 1956, S. C. Cui 118 (PE). Muping Distr.: Yantai, Mt. Kunyuh $\left[37.24^{\circ} \mathrm{N}, 121.78^{\circ} \mathrm{E}\right], 12$ July 1957 , Herbarium of PE 3064 (PE). ShanghaI: Fengxian Distr.: Zhelin Town $\left[30.84^{\circ} \mathrm{N}, 121.48^{\circ} \mathrm{E}\right]$, Sept. $1985, D . X$. Ye 1478 (PE). Shanxi: Pingyao Co.: Montagne NW de Pingyang Pu [mountain NW of Linfen city today] $\left[36.13^{\circ} \mathrm{N}, 111.48^{\circ} \mathrm{E}\right], 12$ July 1915 , s.c. 1455 (PE). Wutai Co.: In monte Ta-Wutai-schan ad confines prov. Tschili [39.014 N, $\left.113.604^{\circ} \mathrm{E}\right], 1929$, J.H. Serre 2285 (W). Sichuan. Cangxi Co.: Gaopo Town, Huabeiliang Hill, alt. $1135 \mathrm{~m}\left[32.08^{\circ} \mathrm{N}, 106.35^{\circ} \mathrm{E}\right], 13$ Aug. 1964 , C. M. He 49 (CDBI). Junlian Co.: Jiefang Township, Taibaishan, alt. $1350-1400 \mathrm{~m}\left[27.92^{\circ} \mathrm{N}, 104.72^{\circ} \mathrm{E}\right]$, 23 July 1964, K. P. Yin \& Q. H. Chen 491 (CDBI). TaIwan. Beitou Distr.: Tatunshan to Taitzupai, near Peitou (Beitou), alt. $700-1000-400 \mathrm{~m}\left[25.18^{\circ} \mathrm{N}\right.$, $\left.121.53^{\circ} \mathrm{E}\right], 8$ July 1969, C. C. Hsu 5486 (S). Nantou Co.: In Monte Taitum $\left[23.930^{\circ} \mathrm{N}, 120.794^{\circ} \mathrm{E}\right], 30$ June 1903 , U. Faurie 752 (W). Yunnan. Funing Co.: Tianpeng, alt. $1540 \mathrm{~m}\left[23.30^{\circ} \mathrm{N}, 105.46^{\circ} \mathrm{E}\right], 13$ June 1964, S. Z. Wang 1014 (KUN). Panlong Distr.: Kunming, Heilongtan $\left[25.14^{\circ} \mathrm{N}, 102.75^{\circ} \mathrm{E}\right], 21$ July 1955 , s.c. 50835 (KUN). ZHEJIANG. Cangnan Co.: Nan Che Shan, ca 70 miles SE of Ping Yung [Pingyang], alt. $125-185 \mathrm{~m}\left[27.629^{\circ} \mathrm{N}\right.$, $\left.120.603^{\circ} \mathrm{E}\right], 25-26$ June 1924, R.C. Ching 1953 (W). Longquan City: Jinx Town, behind the Maoshan Temple, alt. $1110 \mathrm{~m}\left[28.1^{\circ} \mathrm{N}, 118.98^{\circ} \mathrm{E}\right], 27 \mathrm{July} 1958$, R. H. Shan 5546 (KUN). Pingyang Co.: Changqian, Mt. Baiyun $\left[27.55^{\circ} \mathrm{N}, 120.17^{\circ} \mathrm{E}\right], 2$ July 1959 , s.c. 5952 (KUN). Xihu Distr.: Hangzhou, Lingfeng [30.3 $\left.{ }^{\circ} \mathrm{N}, 120.44^{\circ} \mathrm{E}\right]$, 26 June 1957, S. Y. Zhang 1022 (PE); Hangzhou, Yunqi Village $\left[30.19^{\circ} \mathrm{N}, 119.96^{\circ} \mathrm{E}\right], 26$ June 1959 , s.c. 2481 (KUN).Yinzhou Distr.: Ningbo [29.86 $\left.{ }^{\circ} \mathrm{N}, 121.63^{\circ} \mathrm{E}\right]$, 1958, S. Y. Zhang 1085 (PE).

JAPAN. HOKKAIDO IsLAND: Syakotan-gun [Shakotan Distr.], Yobetsu-mura [Mt. Yobetsu] $\left[43.26^{\circ} \mathrm{N}\right.$, $\left.140.54^{\circ} \mathrm{E}\right], 25$ July 1955, M. Furuse s.n. (S); Hakodate $\left[41.762^{\circ} \mathrm{N}, 140.703^{\circ} \mathrm{E}\right], 1861$, Maximowicz s.n. (W); Cote de Nemuro $\left[43.317^{\circ} \mathrm{N}, 145.588^{\circ} \mathrm{E}\right], 1$ Aug. 1889 , s.c. 5039 (W). Honshu IsLand: At the foot of Mt. Fuji, Aokigahara $\left[35.43^{\circ} \mathrm{N}, 138.64^{\circ} \mathrm{E}\right], 5$ Oct. 1961, E. Hultén s.n. (S); Senjyogahara [Senjogahara], Nikko $\left[36.78^{\circ} \mathrm{N}\right.$, $\left.139.45^{\circ} \mathrm{E}\right], 8$ Oct. 1961 , E. Hultén s.n. (S); Shimotakai-gun [Shimo-takai Distr.], vicinity and neighborhood Biwa-ike Shiga-koogen Hirawo-mura $\left[36.74^{\circ} \mathrm{N}\right.$, $138.51^{\circ}$ E], 14 Sept. 1955 , M. Furuse s.n. (S); Shimokita-gun [Shimokita Distr.], for Imooto-numa Horobe Higashi-toori-mura [41.28 $\left.\mathrm{N}, 141.34^{\circ} \mathrm{E}\right], 16$ Sept. 1954 ,
M. Furuse s.n. (S); Honaimura in Echigo $\left[38.06^{\circ} \mathrm{N}\right.$, $\left.139.62^{\circ} \mathrm{E}\right], 18$ July 1954, M. Togasi 1062 (S); KitaTsuru-gun [Kitatsuru Distr.], Seihachi-tooge Sasagomura $\left[35.79^{\circ} \mathrm{N}, 138.91^{\circ} \mathrm{E}\right], 2$ Sept. 1952 , M. Furuse 21965 (S); Kanto region, Agatsuma-gun [Agatsuma Distr.], Iwashima, Agatsuma-machi $\left[36.57^{\circ} \mathrm{N}, 138.82^{\circ} \mathrm{E}\right]$, 25 July 1964, K. Masuda 528 (S); Chôsei-gun [Chosei Distr.], Ichinomiya-chô [Ichinomiya Beach] $\left[35.36^{\circ} \mathrm{N}\right.$, $\left.140.38^{\circ} \mathrm{E}\right]$, 1934, T. Makino s.n. (S); Mt. Chokai, Yurigori [Yurihorijo] $\left[39.19^{\circ} \mathrm{N}, 140.13^{\circ} \mathrm{E}\right.$ ], July 1930 , T. Makino s.n. (S); Kanra-gun [Kanra Distr.], Myôgimachi [Mt. Myôgi] [36.3ํ․ $\left.138.73^{\circ} \mathrm{E}\right], 1920$, T. Makino s.n. (S); Kôza-gun [Koza Distr.], Kugenuma-chô [Kugenuma Beach] $\left[35.32^{\circ} \mathrm{N}, 139.46^{\circ} \mathrm{E}\right], 25$ June 1914 , T. Makino s.n. (S); [Tokyo, Shibuya], Onden [currently Jingumae] near Tokyo $\left[35.67^{\circ} \mathrm{N}, 139.71^{\circ} \mathrm{E}\right], 12$ July 1907 , s.c. s.n. (S). Sizugatake $\left[35.506^{\circ} \mathrm{N}, 136.193^{\circ} \mathrm{E}\right]$, July 1903, U. Faurie 198 (W); Ichinomiya in Kazusa [35.351 $\left.{ }^{\circ} \mathrm{N}, 140.364^{\circ} \mathrm{E}\right], 29$ June 1952, S. Okoyama 536 (W); In monte Futagoyama $\left[36.068^{\circ} \mathrm{N}, 138.863^{\circ} \mathrm{E}\right]$, s.d., J. Matsumura 257 (W). OgasaWARA IsLANDS [first record]: Bonin [Islands] $\left[27.07^{\circ} \mathrm{N}, 142.212^{\circ} \mathrm{E}\right]$, s.d., J. Matsumura 9 (W).

NORTH KOREA. KANGWON. In arenoso littorae Ouen san [39.141 $\left.{ }^{\circ} \mathrm{N}, 127.434^{\circ} \mathrm{E}\right]$, Aug. 1901, U. Faurie 849 (W). Pyongyang. In arenosis Pyeng Yang $\left[39.017^{\circ} \mathrm{N}, 125.676^{\circ} \mathrm{E}\right]$, June 1901, U. Faurie $848(\mathrm{~W})$. RyangGang. Kapsan Distr.: In valle fl. Che-czchen-gan, prope oppidum Kapsan $\left[41.10^{\circ} \mathrm{N}, 128.29^{\circ} \mathrm{E}\right], 18$ July 1897, V. L. Komarov s.n. (LE).

SOUTH KOREA. Seoul City: Seoul $\left[37.57^{\circ} \mathrm{N}\right.$, $\left.126.96^{\circ} \mathrm{E}\right]$, July 1886 , Kalinowsky s.n. (LE). JeOLANAM: Mt. Rokodan [Mt. Rogodan, W part of Jiri Mt., Jiri Mts] [35.294 $\left.{ }^{\circ} \mathrm{N}, 127.531^{\circ} \mathrm{E}\right], 24$ May 1932, M. Boku s.n. (LE).

RUSSIA. Primorsky KraI. Fokino Distr.: Putyatin Island, near Putyatin village $\left[42.86^{\circ} \mathrm{N}, 132.43^{\circ} \mathrm{E}\right]$, 3 Sept. 1982, N. Tzvelev 69 (LE). [Khorolsky Distr.]: Nikolsk-Ussuriysky [currently Ussuriysk], S shore of Khanka Lake, Poluostrov Ryabokon', at the foot of Ryabokon Hill $\left[44.52^{\circ} \mathrm{N}, 132.39^{\circ} \mathrm{E}\right], 15$ Aug. 1924, E. Aintz 3139 (LE). Khasansky Distr.: S of Lake Khanka, 4.5 verst [ca $4.5 \mathrm{~km}$ ] SW of Andreevka [42.639 $\left.{ }^{\circ} \mathrm{N}, 131.133^{\circ} \mathrm{E}\right], 5$ Aug. 1926, G. Melvil s.n. (S). Sakhalin Oblast. Korsakovsky Distr.: Swampy meadow at the seashore near Solovyevka village [46.72 $\left.{ }^{\circ} \mathrm{N}, 142.74^{\circ} \mathrm{E}\right], 18$ Sept. 1982, Tzvelev 283 (LE); In littorae Soriofka [now Solovyevka] $\left[46.716^{\circ} \mathrm{N}\right.$, $\left.142.749^{\circ} \mathrm{E}\right], 2$ Sept. 1908, U. Faurie 801 (W). Dolinsky Distr.: $5 \mathrm{~km}$ NW of Starodupskoye, mouth of Naida [Nayba] River [ $47.44^{\circ} \mathrm{N}, 142.76^{\circ} \mathrm{E}$ ], 2 Oct. 1996, A. V. Buhteeva s.n. (LE). 
VIETNAM [first records]. LaO Kay [LÀo CAI]. Sa Pa Distr.: Chapa [Chapu, Sa Pá], broussailes [thickets], alt. $1500 \mathrm{~m}\left[22.32^{\circ} \mathrm{N}, 103.86^{\circ} \mathrm{E}\right]$, July 1927 , M. Pételot 5080 (P001720033, P001720035).

\section{Calamagrostis tripilifera Hook. f.}

Fl. Brit. India 7(22): 262. 1897[1896].

Basionym: Deyeuxia tripilifera (Hook. f.) Keng, Sunyatsenia 6(1): 68. 1941. LестотуPE (designated by Paszko 2012: 341): INDIA. SiккIM. [North Sikkim Distr.]: Himalaya, Tungu [Thangu] [alt. $c a 3960 \mathrm{~m}$ ] $\left[27.898^{\circ} \mathrm{N}, 88.531^{\circ} \mathrm{E}\right], 1848$, J. D. Hooker s.n. (LECTOTYPE K000098593!).

Deyeuxia flavens Keng, Sunyatsenia 6: 67. 1941. TyPE: CHINA. Gansu. [Xiahe Co.]: Labrang, collected on steppe $\left[35.194^{\circ} \mathrm{N}, 102.504^{\circ} \mathrm{E}\right], 8$ Oct. $1934, C$. W. Yao 515 (p.p., mixed with Poa sphondylodes Trin. var. macerrima Keng) (HOLOTYPE, IBSC, n.v.). Synonymized by Paszko (2012).

Agrostis triaristata auct. non Knapp: Khoi \& Đo, Checkl. Pl. Spec. Vietnam 3: 782. 2005; Tran \& Vu, Proc. 5th Nat. Scien. Conf. Ecol. \& Biol. Res., Hanoi, 18 October 2013, 14. 2013.

Perennial, loosely tufted. Culms erect or geniculate at base, (13-)20-65(-88) cm tall, unbranched, glabrous; nodes 2-4. Sheaths smooth, not inflated; collars smooth; ligules $0.9-6.0 \mathrm{~mm}$ long, membranous, toothed. Cauline blades $2-13(-18) \mathrm{cm}$ long, 1.8-5.3 mm wide, lanceolate-linear, green, flat; midrib not prominent; abaxial surfaces minutely scabrous; adaxial surfaces slightly ribbed, glabrous or minutely scabrid. Panicle open, very loose, 4.5-16.0(-22.5) cm long; branches usually paired, rarely in whorls of 3-4, distant, ascending or widely spreading, the lowest panicle branches $3.0-9.2 \mathrm{~cm}$ long; primary branches glabrous or rarely slightly scabrous, with spikelets clustered in upper half of branch. Spikelets 3.0-7.1 mm long, 1-flowered; glumes subequal, weakly scabrid on keel, acuminate at apex; the lower 3.0-7.1 mm long and 1.15-2.75 mm wide, 1-veined; the upper $2.75-6.40 \mathrm{~mm}$ long and $0.8-1.8 \mathrm{~mm}$ wide, 3-veined, 0.78-0.97 times as long as lower glume; callus hairs $0.65-1.75 \mathrm{~mm}$ long, $0.20-0.48$ times as long as lemmas, surrounding the floret; lemma $2.7-$ $5.5 \mathrm{~mm}$ long, 5-veined, 0.60-1.03 times as long as lower glumes, with dorsal awn 1.75-9.2 mm long, geniculate with twisted column, attached usually on lower third $[0.10-0.25(-0.41)]$ of lemmas, slightly exerted from glumes, lateral and intermediate veins prolonged into $0.20-0.85 \mathrm{~mm}$ long mucros with the outermost mucros longest; palea $1.85-3.80 \mathrm{~mm}$ long, $0.54-0.90$ times as long as lemmas; rachilla extensions $0.3-1.25 \mathrm{~mm}$ long, densely bearded with hairs $1.5-3.0 \mathrm{~mm}$ long. Anthers $0.6-1.5 \mathrm{~mm}$ long. Flowering and fruiting in August and September.

Calamagrostis tripilifera was described by Hooker (1896) from Sikkim (NE India) based on three heterogenous elements. One of them (K000098593!), collected at Tungu (Thangu, N Sikkim), was designated by Paszko (2012) as lectotype. Hooker's $C$. tripilifera was incorrectly placed by Bor (1960) in synonymy with another species, Agrostis triaristata (Hook. f.) Bor, nom. illeg. hom. A recent taxonomic revision (Paszko 2012) revealed that Deyeuxia triaristata and $C$. tripilifera represent two different species, C. tripilifera and C. filiformis Griseb. (with Deyeuxia triaristata in synonymy with the latter). Paszko (2012) synonymized Chinese Deyeuxia flavens Keng within C. tripilifera. Calamagrostis tripilifera differs from $C$. filiformis by having shorter mucros at the lemma tip (0.20-0.85 vs. 0.90-1.75 mm long), broader lower glumes (1.152.75 vs. $0.9-1.5 \mathrm{~mm}$ wide), a higher ratio of lower glume width to lower glume length $(0.25-0.51$ vs. $0.19-0.32$ ) and a lower ratio of upper to lower glume length (0.8-0.9 vs. 0.9-1.0) (Paszko 2012).

Paszko (2012) did not revise the Vietnamese collection of C. tripilifera recorded by Camus (1928) and subsequently by Schmid (1958). Our study confirms its correct identification made by Camus (1928), who recorded it from Mount Fan Si Pan (Fansipan), the highest point in Indochina (3143 m). Mount Fan Si Pan is situated on the border between Lai Chau and Lao Cai Provinces, $9 \mathrm{~km}$ southwest of $\mathrm{Sa} \mathrm{Pa}$, a Vietnamese mountain health resort.

Habitat and Phenology. Perennial, loosely tufted. Calamagrostis tripilifera occurs in subalpine and alpine meadows, grassy slopes, open woodlands or shrublands. $2700-4900 \mathrm{~m}$. Flowering July-September. 
General Distribution. China (N Chongqing Municipality, Gansu, Sichuan, Qinghai, Xizang and Yunnan Provinces), India (N Sikkim Province) and NW Vietnam (Lao Kay Province) (Fig. 3).

Notes. Most of the localities of $C$. tripilifera are in the eastern part of the Qinghai-Xizang (Tibetan) Plateau in China, where it is quite common in subalpine and alpine zones. It also has scattered localities in adjacent regions such as the Sikkim Himalayas (NE India), Daba Mts (Chongqing, Southwest China), Jiaozishan Mts (Yunnan, Southwest China) and Hoan Lien Son Mts (NW Vietnam), where it can occur occasionally in the subalpine and alpine zones of these mountains. Calamagrostis tripilifera ranges as far west as northern Sikkim (NE India) on the southern slopes of the Eastern Himalayas. It has also been recorded from the northern side of that range at a few scattered localities in adjacent parts of the Chinese Xizang Autonomous Region (Tibet), in Damxung and Mainling Counties. It is known from one locality in northern Vietnam. It was recorded there by Schmid (1958) from Mount Fan Si Pan. Its occurrence in northwestern Vietnam represents the southern limit of its known geographical range.

In Flora of China, Lu et al. (2006) recorded Calamagrostis tripilifera (as Deyeuxia flavens) only from Gansu, Qinghai, Sichuan, Xizang and Yunnan Provinces. Here we provide its first record from Chongqing Municipality, created on 14 March 1997; formerly it was part of Sichuan Province, China. Calamagrostis tripilifera was collected in the Hongchiba National Forest Park located in Wuxi County, in the Daba Mts region; the Daba Mts are part of the larger Qinling Range in the northern part of Chongqing Municipality. The occurrence of $C$. tripilifera in the Daba Mts represents the eastern limit of its known geographical range.

SPeCimens EXAmined: CHINA. ChONGQING [first record]. Wuxi Co.: [Daba Mts], Hongchiba [National Forest Park in the vicinity of Wenfengzhen] $\left[31.414^{\circ} \mathrm{N}\right.$, $\left.109.232^{\circ} \mathrm{E}\right], 18$ July 1988, Y. H. Liu Wild-Experiment 8 (PE). GANsu. Bairi Co.: s.l. [36.974 $\left.\mathrm{N}, 103.133^{\circ} \mathrm{E}\right]$, 13 Sept. 1957, J. B. Yong 251 (PE); Lanzhou City: s.l. $\left[36.069^{\circ} \mathrm{N}, 103.751^{\circ} \mathrm{E}\right], 23$ Aug. 1956 , s.c. 22 (PE). Luqu
Co.: Luqu, ca 30-40 km N of Gansu-Sichuan boarder on road from Chengdu to Lanzhou, $c a 20 \mathrm{~km} \mathrm{~S}$ of Waxu and $10 \mathrm{~km}$ E of Gahai, $34.167^{\circ} \mathrm{N}, 102.4167^{\circ} \mathrm{E}$, alt. $3440 \mathrm{~m}$, 18 Sept. 1997, R. J. Soreng et al. 5385 (US). Maqu Co.: s.1., $33.995^{\circ} \mathrm{N}, 102.077^{\circ} \mathrm{E}, 15$ Aug. $1956, H$. D. Wang 1803-1 (PE). Xiahe Co.: Labuleng [Labrang] Temple, alt. $2980 \mathrm{~m}, 35.194^{\circ} \mathrm{N}, 102.504^{\circ} \mathrm{E}, 25$ July 1999, W. L. Chen 11 (PE); Ganping Temple $\left[35.313^{\circ} \mathrm{N}\right.$, $\left.102.284^{\circ} \mathrm{E}\right]$, s.d., Y. L. Keng \& P. C. Keng 5713 (PE, 2 sheets). Yongdeng Co.: Ta Hwa, near Pingfan [now Yongdeng] [ $36.729^{\circ} \mathrm{N}, 103.242^{\circ} \mathrm{E}$ ], alt. 2900 to $3100 \mathrm{~m}$, 17 July 1923, R. C. Ching $521 a$ (US). QinghaI. Datong Co.: Niuchang, Hongyahe [36.93 $\left.{ }^{\circ} \mathrm{N}, 101.689^{\circ} \mathrm{E}\right]$, 21 Aug. 1957, P. C. Keng et al. 160 (PE, 2 sheets). Gangca Co.: Rexiaozhakuang, alt. $3640 \mathrm{~m}\left[37.327^{\circ} \mathrm{N}\right.$, $\left.100.132^{\circ} \mathrm{E}\right], 15$ Aug. 1972, J. W. Zhang \& S. Z. Sun 248 (PE). Gui'de Co.: Lajishan Mts, road S of Lajishan Mts, subalpine vegetation with Salix and Rhododendron species, $36.301^{\circ} \mathrm{N}, 101.629^{\circ} \mathrm{E}$, alt. $3340 \mathrm{~m}, 1$ Aug. 2010 , B. Paszko GPS 582 (KRAM). Henan Co.: Keduo Xiang, $3400 \mathrm{~m}$, growing among shrubs on $\mathrm{N}$ slope, $3400 \mathrm{~m}$, $34.738^{\circ} \mathrm{N}, 101.613^{\circ} \mathrm{E}, 19$ Sept. 1970, S. $-W$. Liu 1890 (CAS). Menyuan Co.: Shangwulan, roadside $\left[37.374^{\circ} \mathrm{N}\right.$, $\left.101.615^{\circ} \mathrm{E}\right], 16$ Aug. 1957, P. C. Keng et al. 81 (PE). Nangqên Co.: Juela, Nasuji, meadow on shady slope, alt. $4300 \mathrm{~m}\left[32.207^{\circ} \mathrm{N}, 96.478^{\circ} \mathrm{E}\right], 20$ Aug. 1965, Y. C. Yang 1355 (KUN). Qilian Co.: Mole, river side, alt. $3650 \mathrm{~m}$ [38.175 $\left.{ }^{\circ} \mathrm{N}, 100.24^{\circ} \mathrm{E}\right], 12$ Aug. 1958, P. C. Tsoong 8693 (PE, 2 sheets). Yushu City: just E of Jiangxi Forest Station on E side of $\mathrm{Zi} \mathrm{Qu}, \mathrm{SE}$ of Mozhong, growing under Picea, alt. $3540 \mathrm{~m}, 34.0833^{\circ} \mathrm{N}, 97.017^{\circ} \mathrm{E}, 27 \mathrm{Aug}$. 1996, T. N. Ho et al. 2454 (GH, 3 sheets). Zadoi Co.: Sulu, Banayong, meadow on slope, alt. 4200-4400 m [32.902 ${ }^{\circ}$ N, $\left.95.301^{\circ} \mathrm{E}\right], 11$ Aug. 1965, S. W. Liu 372 (PE). Zêkog Co.: Duofudun Xiang, growing under bushes, alt. $3400 \mathrm{~m}\left[35.037^{\circ} \mathrm{N}, 101.471^{\circ} \mathrm{E}\right], 22$ Aug. 1970, S. W. Liu 1564 (CAS). Sichuan. Baiyü Co.: Pedigree station of Changtai, Gantongba, alpine meadow, alt. $3950 \mathrm{~m}\left[31.217^{\circ} \mathrm{N}, 98.827^{\circ} \mathrm{E}\right], 23$ Aug. 1982, Z. X. Tang et al. 786 (PE); Nata, Paguo, alpine meadow, alt. $4200 \mathrm{~m}\left[31.217^{\circ} \mathrm{N}, 98.827^{\circ} \mathrm{E}\right], 16$ Aug. $1982, Z$. X. Tang et al. 754 (PE, 2 sheets); Changtai, Shutuo $\left[31.217^{\circ} \mathrm{N}\right.$, $\left.98.827^{\circ} \mathrm{E}\right], 18$ Aug. 1982, X. W. Tian 118-6 (PE). Batang Co.: W slope of Mt. Haizi, alt. $4670 \mathrm{~m}\left[30.012^{\circ} \mathrm{N}\right.$, $\left.99.114^{\circ} \mathrm{E}\right], 29$ July 1983, K. Y. Lang et al. 2507 (KUN). Daocheng Co.: Midway from Yading to Daocheng, alt. $3700 \mathrm{~m}\left[29.037^{\circ} \mathrm{N}, 100.297^{\circ} \mathrm{E}\right], 18$ Sept. 2002, H.Y. Ma 90 (KUN). Dawu Co.: Yuke, Qimei, alpine meadow, alt. $4300 \mathrm{~m}\left[30.989^{\circ} \mathrm{N}, 101.122^{\circ} \mathrm{E}\right], 15$ Sept. 1974 , s.c. 5986 (CDBI, PE); Zhongzhi(?), alpine meadow, alt. $4500 \mathrm{~m}$ [30.989 ${ }^{\circ}$, $\left.101.122^{\circ} \mathrm{E}\right], 17$ Aug. 1974, Y. G. Liu 7618 (CDBI, PE). Dêgê Co.: Near Cuodeng Bridge, grassland, 


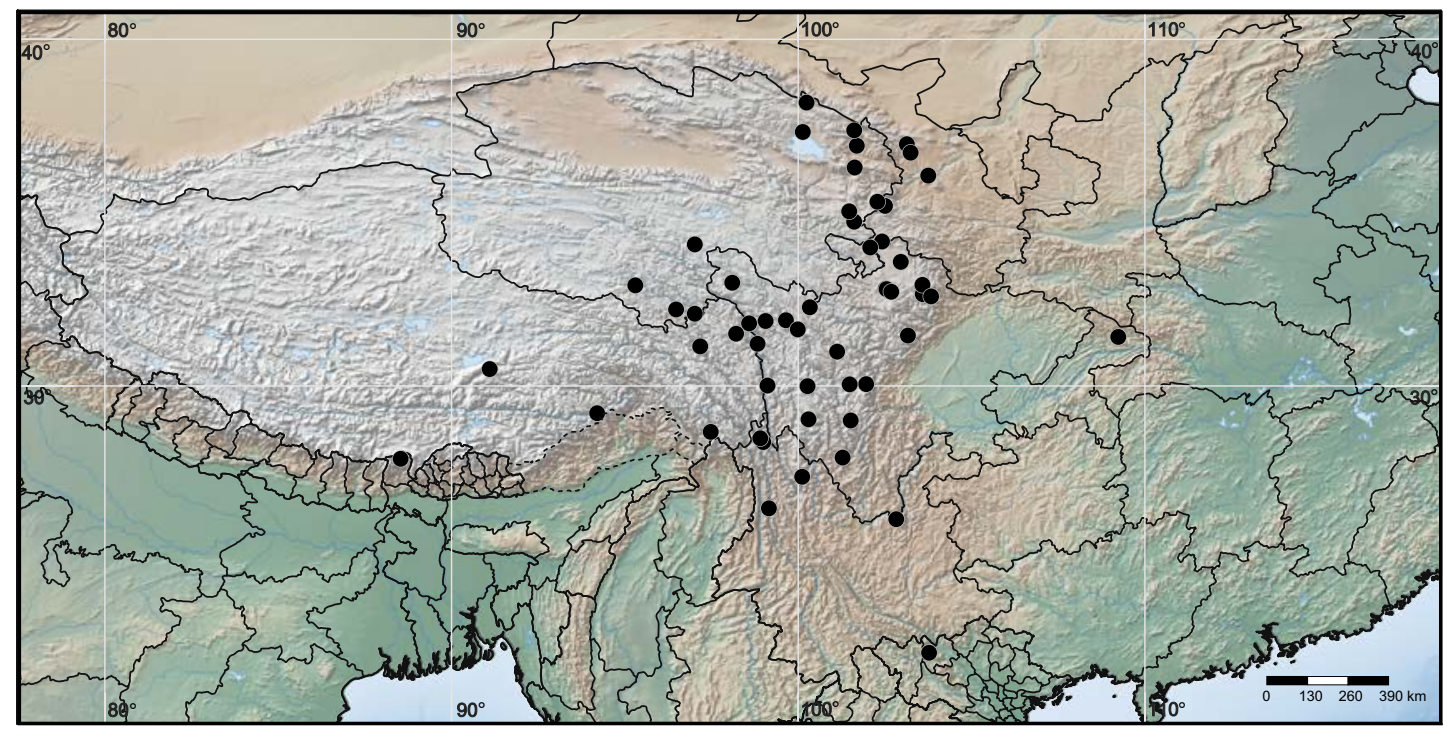

Fig. 3. General distribution of Calamagrostis tripilifera Hook. f.

$4000 \mathrm{~m}\left[31.805^{\circ} \mathrm{N}, 98.584^{\circ} \mathrm{E}\right], 12$ July 1961 , SouthNorth Water Transfer Project Team 9341 (PE); Mawei(?), subalpine meadow, alt. $3960 \mathrm{~m}\left[31.805^{\circ} \mathrm{N}, 98.584^{\circ} \mathrm{E}\right]$, 7 Aug. 1982, X. W. Tian 68(11) (PE); Manigange, alpine meadow, $3960 \mathrm{~m}\left[31.80517^{\circ} \mathrm{N}, 98.583702^{\circ} \mathrm{E}\right]$, 7 Aug. 1982, X. W. Tian 68(11) (PE); N slope of Que'er Mt., alt. $3900-4000 \mathrm{~m}\left[31.873^{\circ} \mathrm{N}, 99.059^{\circ} \mathrm{E}\right], 9$ Aug. 1983 , F. B. Wang 83225 (PE). Garzê Co.: Datangba, Zhekehe [Zhakexiang], alt. $4000 \mathrm{~m}\left[31.895^{\circ} \mathrm{N}, 99.645^{\circ} \mathrm{E}\right]$, 30 Aug. 1982, X. W. Tian 145-8 (PE); Datangba, alt. $4200 \mathrm{~m}\left[31.631^{\circ} \mathrm{N}, 99.987^{\circ} \mathrm{E}\right], 30$ Aug. 1982 , Tian et al. 147 suppl. (PE); Datangba, Lina, alpine meadow, alt. $4350 \mathrm{~m}\left[31.631^{\circ} \mathrm{N}, 99.987^{\circ} \mathrm{E}\right], 30 \mathrm{Aug} .1982, X$. W. Tian 148-5 (PE); Tangke Ranch, new linen factory, grassland, alt. 3400 m, 18 Aug. 1963, X. Q. Li 390 (CDBI). Hongyuan Co.: $2 \mathrm{~km}$ NW of county town, semimarsh grassland, alt. $3400 \mathrm{~m}\left[32.803^{\circ} \mathrm{N}, 102.524^{\circ} \mathrm{E}\right]$, 19 Aug. 1983, Z. X. Tang 1768 (PE, 2 sheets); Amukehe, Nilongqinba(?), grassland [ $32.793^{\circ} \mathrm{N}, 102.549^{\circ} \mathrm{E}$, 30 July 1964, B. Z. Ni 312 (CDBI, PE); Jiulong Co.: $6 \mathrm{~km} \mathrm{~N}$ of Jichou Valley, among alpine bushes on slope $\left[29.004^{\circ} \mathrm{N}, 101.514^{\circ} \mathrm{E}\right], 8$ July 1982 , Z. X. Tang et al. 243 (PE). Kangding Co.: Tsipula [pass], in prato subhumido, alt. $3600 \mathrm{~m}\left[32.717^{\circ} \mathrm{N}, 102.683^{\circ} \mathrm{E}\right], 2 \mathrm{Aug}$. 1922, H. Smith 4152 (PE); Zheduo Mt., grassland on slope, alt. $4400 \mathrm{~m}\left[30.052^{\circ} \mathrm{N}, 101.96^{\circ} \mathrm{E}\right], 30$ July 1963 , $W$ Sichuan Team 1153 (PE, 2 sheets); Zheduo Mt. $\left[30.052^{\circ} \mathrm{N}, 101.96^{\circ} \mathrm{E}\right], 21$ July 1954, Z. L. Wu 40854 (PE); Xinduqiao, alt. $3800 \mathrm{~m}\left[30.046^{\circ} \mathrm{N}, 101.4842^{\circ} \mathrm{E}\right]$, 3 Sept. 1980, T. C. Mi 29651 (CDBI); Gao'ershi, slope, alt. $3400 \mathrm{~m}\left[30.052^{\circ} \mathrm{N}, 101.96^{\circ} \mathrm{E}\right], 27$ Aug. 1982, $W$. Hou 29521 (CDBI); Tagong, among alpine bushes, alt. $3110 \mathrm{~m}\left[30.046^{\circ} \mathrm{N}, 101.484^{\circ} \mathrm{E}\right], 1$ Sept. 1979, B. $Z$. Qiu 21092 (CDBI). Li Co.: Zhegu Mt., alt. $3600 \mathrm{~m}$ [31.454 $\left.{ }^{\circ} \mathrm{N}, 103.164^{\circ} \mathrm{E}\right], 4$ Sept. 1957, X. Li \& J. X. Zhou 74430 (PE); Zhegu Mt., Shanglu, alt. $3650 \mathrm{~m}$ [31.454 ${ }^{\circ}$ N, $\left.103.164^{\circ} \mathrm{E}\right], 2$ Sept. 1957, X. Li 74348 (PE). Litang Co.: SW of Maoyaba, alpine semi-marsh grassland, alt. $4150 \mathrm{~m}\left[29.993^{\circ} \mathrm{N}, 100.268^{\circ} \mathrm{E}\right], 26$ July 1982, Z. X. Tang et al. 512 (PE); SW of Maoyaba, alpine semi-marsh grassland, alt. $4150 \mathrm{~m}\left[29.993^{\circ} \mathrm{N}\right.$, $100.268^{\circ} \mathrm{N}$ ], 26 July 1982, Z. X. Tang et al. 512 (PE, 2 sheets). Mili Co.: Sanqu to Ba'er Pasture, under Rhododendron, alt. $3000 \mathrm{~m}\left[27.934^{\circ} \mathrm{N}, 101.28^{\circ} \mathrm{E}, 13\right.$ Sept. 1983, Qinghai-Tibetan Team 14765 (KUN). Sêrtar Co.: Nianlong Temple, Selacuo Valley, meadow in valley, alt. $4200 \mathrm{~m}\left[32.27^{\circ} \mathrm{N}, 100.335^{\circ} \mathrm{E}\right], 27$ July $1974, D . H . W u$ Se 6673 (PE). Sêrxü Co.: Shebei Mt., roadside on sunny slope, alt. $3900 \mathrm{~m}\left[32.977^{\circ} \mathrm{N}, 98.097^{\circ} \mathrm{E}\right], 31 \mathrm{Aug} .1974$, J. Q. Wang 4951 (PE). Shiqu Co.: Gucaoma, slope, alt. $4280 \mathrm{~m}\left[32.977^{\circ} \mathrm{N}, 98.097^{\circ} \mathrm{E}\right], 09$ Aug. 1980 , s.c. 28358 (CDBI). Songpan Co.: Duolitai, semi-marsh grassland, alt. $3800 \mathrm{~m}\left[32.648^{\circ} \mathrm{N}, 103.593^{\circ} \mathrm{E}\right], 11$ Aug. 1983, Z. X. Tang 1700 (PE); Chuanzhusi Town, Huang-chienkuan (Huangshengguan), in mixed forest, alt. $3300 \mathrm{~m}$ [32.917 N, $\left.103.583^{\circ} \mathrm{E}\right], 29$ Aug. 1922, H. Smith 4059 (PE); Dongrergo [Hsioeh-pao-ting, Hsueh-po-ting], Huang-lung-ssii [Huanglongsi], alt. 4800-5000 m $\left[32.583^{\circ} \mathrm{N}, 103.833^{\circ} \mathrm{E}\right], 21$ July $1922, H$. Smith 3288 (PE). Zoigê Co.: Leimanzhong, Yangfangqian, alt. 
$3440 \mathrm{~m}\left[33.579^{\circ} \mathrm{N}, 102.958^{\circ} \mathrm{E}\right], 4$ Aug. 1975 , s.c. 10352 (PE); Xiaman, Zhongyangfang, alt. $3440 \mathrm{~m}\left[33.579^{\circ} \mathrm{N}\right.$, $\left.102.958^{\circ} \mathrm{E}\right], 4$ Aug. 1975 , s.c. 10352 (CDBI); Tangke, alt. $3440 \mathrm{~m}\left[33.579^{\circ} \mathrm{N}, 102.958^{\circ} \mathrm{E}\right], 8$ Aug. 1975 , s.c. 10376 (PE). Xizang. Damxung Co.: Weiyuanduo $\left[30.487^{\circ} \mathrm{N}, 91.099^{\circ} \mathrm{E}\right], 11$ Aug. 1985 , s.c. 3033 (PE). Jomda Co.: Ela Mt., among Rhododendron bushes, alt. $4320 \mathrm{~m}\left[31.506^{\circ} \mathrm{N}, 98.208^{\circ} \mathrm{E}\right], 26$ Aug. 1976, QinghaiTibetan Ecology Team 9832 (PE). Mainling Co.: Pai Town, slope $\left[29.214^{\circ} \mathrm{N}, 94.199^{\circ} \mathrm{E}\right], 17$ Aug. 1981, Qinghai-Tibetan Team 1081 (PE). Qamdo Co.: Tuoba, alt. 3900-4000 m, grassland near Picea forest, alt.

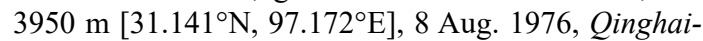
Tibetan Team 12653 (PE). Zayü Co.: Demula Mt., meadow, alt. $3000 \mathrm{~m}\left[28.675^{\circ} \mathrm{N}, 97.476^{\circ} \mathrm{E}\right], 29$ Aug. 1976, Wu et al. 5212 (KUN). Yunnan. Dêqên Co.: Baimang Mt., among alpine bushes, alt. 4200-4300 m [28.381 $\left.{ }^{\circ} \mathrm{N}, 98.99^{\circ} \mathrm{E}\right], 18$ Aug. 1981, Hengduan Mt. Team 3293 (PE); 122 Road Class [28.489 ${ }^{\circ}$, $98.905^{\circ} \mathrm{E}$ ], 24 Aug. 1981, B. S. Li s.n. (PE). Fugong Co.: Biluo Mt., Jiumingfang, under Rhododendron on slope, alt. $4200 \mathrm{~m}$ [26.47 $\left.{ }^{\circ} \mathrm{N}, 99.154^{\circ} \mathrm{E}\right], 14$ Sept. 1964, S. K. Wu 8890 (KUN). Luquan Co.: Wumeng Town, top of Jiaozishan, on meadow or in rock crevice, alt. $4223 \mathrm{~m}, 26.157^{\circ} \mathrm{N}$, 102.828 E, 3 Aug. 2008, H. Peng et al. 9427 (KUN); Wumeng Town, top of Jiaozishan, alpine meadow, alt. $4223 \mathrm{~m}, 26.157^{\circ} \mathrm{N}, 102.828^{\circ} \mathrm{E}, 3$ Aug. $2008, H$. Peng et al. $9444 B$ (KUN). Shangri-La City: Haba Mt., under forest, alt. $2700 \mathrm{~m}\left[27.380^{\circ} \mathrm{N}, 100.115^{\circ} \mathrm{E}\right], 27$ Sept. 2002, H. Y. Ma 119 (KUN).

VIETNAM. Lao Kay (Lào Cai): Massif du Fan Tsi Pan [Phan Xi Păng, Fansipan], Chapu [Chapa, Sa Pá, Sapa], alt. $2900 \mathrm{~m}\left[22.303^{\circ} \mathrm{N}, 103.775^{\circ} \mathrm{E}\right]$, Aug. 1942, Petelot 7988 (P02651396, P03252805).

Notes. Three species of Calamagrostis (C. abnormis, $C$. extremiorientalis and $C$. tripilifera) are recorded in the northwestern part of Vietnam. They are considered indigenous to this area. Their main distribution ranges are in China (Figs 1-3).

The geobotanical division of China and adjacent areas has not yet been settled, and various authors determine it differently. According to Chinese phytogeography, most regions of China are located within the Eastern Asiatic Floristic Kingdom, which can be further divided into three subkingdoms: $(i)$ the Qinghai-Xizang (Tibetan) Plateau floristic subkingdom, (ii) the Sino-Himalayan Forest floristic subkingdom and (iii) the Sino-Japanese Forest floristic subkingdom (Wu \& Wu 1998; Sun 2013).
The territory of Vietnam, lying northwest of the Song Hong River, represents the southern extension of the Himalayas (Averyanov et al. 2003). The other name of the Vietnamese Hoang Lien Son Range, part of the larger Ailao Shan Range, is the Tail of the Himalayas. Phytogeographically, according to Averyanov et al. (2003) the highland regions of the Vietnamese Lai Chau, Lao Cai and Yen Bai administrative provinces lie within the Sikang-Yunnan floristic province [= Sino-Himalayan Forest subkingdom of $\mathrm{Wu}$ and $\mathrm{Wu}$ (1998) and Sun (2013)]. The largest part of this province lies within China and includes the Eastern Himalayas, Hengduan Mt. and the Yunnan Plateau proposed by $\mathrm{Wu}$ and $\mathrm{Wu}$ (1998) in their preliminary floristic division of China. The Khasi-Manipur and northern Burma (N Myanmar) regions were omitted by $\mathrm{Wu}$ and $\mathrm{Wu}$ (1998), but they mentioned that according to Takhtajan's (1986) description they are also part of the Sino-Himalayan Forest subkingdom.

Considering the known distribution patterns of C. abnormis, C. extremiorientalis and C. tripilifera (Figs 1-3), they all are confined to the East Asiatic region. Three geographical elements can be recognized, because they are more or less restricted to one phytogeographical subregion. Calamagrostis extremiorientalis is confined to the Sino-Japanese subregion, C. abnormis to the Sino-Himalayan subregion, and C. tripilifera to the Qinghai-Xizang (Tibetan) Plateau subregion.

ACKnowledgements. We are grateful to Dr. Jeffery M. Saarela (Canadian Museum of Nature, Ottawa) and Dr. Liu Su (Chenshan Botanical Garden, Shanghai) for helpful remarks and suggestions on the manuscript, and to the Curators of CAS, GH, KRAM, L, NEFI, TI and US for loans of their collections. Research visits of Beata Paszko to herbaria in Beijing (PE), Chengdu (CDBI), Kunming (KUN), Sankt Petersburgh (LE) and Stockholm (S) were supported by the exchange programs between the Polish Academy of Sciences and counterpart National Academies, and to Edinburgh (E), London (BM, K), Paris (P) and Vienna (W) by SYNTHESYS Projects (grant nos. FR-TAF-6137, GB-TAF-2262, GB-TAF-4900, AT-TAF-6826) financed by the European Community Research Infrastructure Action under the FP7 'Capacities' Programme. This study was also financed in part from the statutory fund of the W. Szafer Institute of Botany of the Polish Academy of Sciences. 


\section{REFERENCES}

ANONYmous 2017. Oceanic islands plant specimen database. [15 April 2017]. http://umdb.um.u-tokyo.ac.jp/fmi/xsl/ DShokubu/eng_spm/top.xsl.

Averyanov L. V., Phan K. L., Nguyen T. H., Harder D. K. 2003. Phytogeographic review of Vietnam and adjacent areas of Eastern Indochina. Komarovia 3: 1-83.

Bor N. L. 1954. Notes on Asiatic grasses: XVII. The species Agrostis zenkeri Trin. Kew Bull. 9: 441-442.

Bor N. L. 1960. Grasses of Burma, Ceylon, India and Pakistan (excluding Bambuseae). Pergamon Press, London.

CAmus A. 1928. Quelques Graminées nouvelles pour la flore de 1'Indo-Chine. Bull. Soc. Bot. France 75: 552-555.

Handel-Mazzetti H. F. 1936. Botanische Ergebnisse der Expedition der Akademie der Wisssenschaften in Wien nach Südwest-China 1914-1918. VII. Anthophyta. Symb. Sin. 7: 731-1450.

Handel-Mazzetti H. 1996. A botanical pioneer in south west China. Translated by David Winstanley from the 1927 edition Naturbilder aus Sudwest China. http://www.paeo.de/ h1/hand_maz/pioneer/buch.html.

Herner G. 1988. Harry Smith in China: routes of his botanical travels. Taxon 37(2): 299-308.

Hiтchсоск A. S. 1934. New species of Aulacolepis and other grasses. J. Wash. Acad. Sci. 24(7): 290-292.

Hooker J. D. 1847-1851. Letter from Hooker, Sir Joseph Dalton, to unknown addressee, from Sikkim. [1851]. JDH/1/10 f.328-329. Indian Letters 1847-1851. Library, Art, \& Archives Collections, Royal Botanic Gardens, Kew, UK. http://www.kew.org/learn/library-art-archives/ joseph-hooker/letter-hooker-sir-joseph-dalton-unknownaddressee-sikkim

HoOKer J. D. 1854. Himalayan Journals, or notes of a naturalist in Bengal, the Sikkim and Nepal Himalayas, the Khasia Mountains, \& c. 1 \& 2. J. Murray, London.

Hooker J. D. 1896. Flora of British India 7. L. Reeve \& Co., London.

Kнor N. K. \& Đo N. T. 2005. Agrostis, Calamagrostis \& Deyeuxia. In: N. T. BAN (ed.), Danh Luc Cac Loai Thuc Vat Viet Nam [Checklist of Plant Species in Vietnam] 3. Nganh Moc Lan - Magnoliophyta (Nganh Hat Kin-Angiospermae), pp. 774, 782. Agricultural Publishing House: Hanoi, Vietnam (in Vietnamese).

KINGDON-WARD F. 1936. Botanical and geographical explorations in Tibet, 1935. Geogr. J. 88(5): 385-410.

KINGDON-WARD F. 1940. Botanical and geographical explorations in the Assam Himalaya. Geogr. J. 96(1): 1-13.

Korthof H. M. \& VeldKamp J. F. 1984. A revision of Aniselytron with some new combinations in Deyeuxia in SE Asia (Gramineae). Gard. Bull. Singapore 37: 213-223.
Lu S. L., Chen W. L. \& Phillips S. M. 2006. Deyeuxia. In: Z. Y. Wu, P. H. RAVEN \& D. Y. Hong (eds), Flora of China: Poaceae 22: 348-359. Science Press, Beijing \& Missouri Botanical Garden Press, St. Louis.

Nobis M., Nowak A., Piwowarczyk R., Ebel A. L., Király G., Kushunina M., Sukhorukov A. P., Chernova O. D., Kipriyanova L. M., Paszko B., Seregin A. P., ZalewskaGalosz J., Denysenko M., Nejfeld P., Stebel A. \& GudKovA P. D. 2016. Contribution to the flora of Asian and European countries: new national and regional vascular plant records, 5. Botany Letters 163(2): 159-174.

Noltie H. J. 1999. Notes relating to the Flora of Bhutan: XXXIX. Gramineae II. Edinburgh J. Bot. 56: 381-404.

Noltie H. J. 2000. Flora of Bhutan 3(2). The Grasses of Bhutan. Royal Botanic Garden Edinburgh \& Royal Government of Bhutan, Edinburgh.

Ohi-Toma T., Watanabe-Toma K. \& Murata J. 2015. Typification of Angiospermae described from the Bonin Islands 2: Archichlamydeae. Phytotaxa 227(1): 35-44.

Paszko B. 2012. Taxonomic revision of Calamagrostis filiformis, C. tripilifera and their allies (Poaceae: Agrostidinae). Polish Bot. J. 57(2): 335-346.

Paszko B. 2014. Deyeuxia himalaica (Poaceae, Agrostidinae): taxonomy and its first record from Myanmar. Phytotaxa 156(5): 285-290.

PAszKo B. 2015. The first record of the Sino-Himalayan species Deyeuxia himalaica in the Yunnan Province, SW China, and three new combinations in Calamagrostis (Poaceae, Agrostidinae). Polish Bot. J. 60(2): 141-145.

PaszKo B. 2016. Calamagrostis nyingchiensis, a new combination for Deyeuxia nyingchiensis (Poaceae, Agrostidinae), and its first record from Yunnan Province, SW China. Polish Bot. J. 61(1): 53-57.

Paszko B. \& LiU B. 2016. First confirmed records of Agrostis scabra (Poaceae, Agrostidinae) in the Chinese flora. Polish Bot. J. 62(2): 253-256.

Paszko B. \& Ma H. Y. 2011. Taxonomic revision of the Calamagrostis epigeios complex with particular reference to China. J. Syst. Evol. 49(5): 495-504.

Paszko B. \& Soreng R. J. 2013. Species delimitation and name application in Deyeuxia abnormis, Agrostis zenkeri, A. pleiophylla and related taxa (Poaceae: Agrostidinae). Phytotaxa 111(1): 1-26.

Paszko B., Chen W. L. \& Liu B. 2016a. Calamagrostis altaica, a neglected species of the Chinese Flora, and note on C. korotkyi (Poaceae, Agrostidinae). Phytotaxa 286(4): 256-266.

Paszko B., Chen W. L. \& LiU B. 2016b. Confirmation of Calamagrostis salina in China, previously misidentified as C. macilenta, and notes about C. kokonorica and C. macilenta (Poaceae, Agrostidinae). Phytotaxa 268(4): 251-262.

Paszko B., Chen W. L. \& Szczepaniak M. 2013. Deyeuxia debilis (Poaceae, Agrostidinae): typification, taxonomy and update of the Chinese distribution. Phytotaxa 135(1): 1-10. 
Probatova N. S. 1985. Poaceae. In: S. S. Kharkevich (ed.), Sosudistye rasteniya Sovetskogo Dalnego Vostoka 1: 89-382. Nauka, Leningrad (in Russian).

Saarela J. M., Liu Q., Peterson P. M., Soreng R. J. \& Paszko B. 2010. Phylogenetics of the grass 'Aveneaetype plastid DNA clade' (Poaceae: Pooideae, Poeae) based on plastid and nuclear ribosomal DNA sequence data. In: O. Seberg, G. Petersen, A. Barfod \& J. I. Davis (eds), Diversity, phylogeny, and evolution in the monocotyledons, pp. 557-587. Aarhus University Press, Aarhus.

Schmid M. 1958. Flore agrostologique de l'Indochine. Agron. Trop. (Nogent-sur-Marne) 13: 304-309.

Sun H. 2013. Phytogeographical regions of China. In: D. Y. Hong \& S. Blackmore (eds), Plants of China: A companion to the Flora of China, pp. 176-204. Science Press, Beijing.

TAKHTAJAn A. 1986. Floristic regions of the World. University of California Press, Berkeley.

THIERS B. 2016 [continuously updated]. Index herbariorum: a global directory of public herbaria and associated staff.
New York Botanical Garden's virtual herbarium. [15 Aug. 2016]. http://sweetgum.nybg.org/science/ih/.

Tran T. P. A. \& Vu T. C. 2013. Tribus Aveneae (Poaceae) for the flora of Vietnam. Proceedings of the 5th National Scientific Conference on Ecology and Biological Resources, Hanoi, 18 October 2013, pp. 11-15. Institute of Ecology and Biological Resources, Hanoi, Vietnam.

TzVelev N. N. 1965. De genere Calamagrostis Adans. in URSS notulae systematicae. Novosti Sist. Vyssh. Rast. 2: 5-50 (in Russian).

TzVelev N. N. 1976. Zlaki SSSR. Nauka, Leningrad.

VLASENKo P. 2008. Soviet military topographic maps 1:200.000. [February 2016]. http://maps.vlasenko.net/soviet-militarytopographic-map/map200k.html.

Wu Z. Y. \& Wu S. G. 1998. A proposal for a new floristic kingdom (realm): the E. Asiatic Kingdom, its delineation and characteristics. In: A. L. ZHANG \& S. G. WU (eds), Floristic characteristics and diversity of East Asian plants, pp. 3-42. China Higher Education Press, Beijing \& Springer Verlag, Berlin. 